\title{
Neuer Schlauch für alten Wein? Eine konzeptuelle Betrachtung von individueller Förderung im Unterricht
}

\section{Hanna Dumont}

Online publiziert: 2. August 2018

(C) Der/die Autor(en) 2018

Zusammenfassung Der vorliegende Beitrag betrachtet den in Politik und Praxis populären Begriff der individuellen Förderung. Dazu wird zunächst erörtert, in welchen Kontexten und in welcher Form der Begriff bislang verwendet wurde. Anschließend werden im wissenschaftlichen Diskurs etablierte verwandte Konzepte von individueller Förderung und Erkenntnisse aus der kognitiven Lehr-Lern-Forschung und der Unterrichtsforschung dargestellt. Darauf aufbauend wird vorgeschlagen, individuelle Förderung im Sinne einer tiefgreifenden Gesamtunterrichtsstrategie zu verstehen, bei der die Lehrkraft den einzelnen Schülerinnen und Schülern ein an ihre spezifischen Lernvoraussetzungen angepasstes Lernangebot macht und das Ziel einer kontinuierlich zunehmenden Verantwortungsübernahme der Schülerinnen und Schüler für ihren eigenen Lernprozess verfolgt. Abschließend werden Implikationen dieser Konzeption für die Schulpraxis und die Bildungsforschung diskutiert.

Schlüsselwörter Individuelle Förderung · Adaptivität · Unterricht · Lehr-LernForschung

\section{Adaptive teaching: Conceptual reflections}

Abstract The present paper deals with the German concept "Individuelle Förderung", which has been widely used in both policy and practice in recent years and may best be translated into "adaptive teaching" or "individualized instruction". The paper first addresses the contexts and ways in which the term has been used. Several related

Der folgende Beitrag ist im Rahmen des „Jacobs Foundation Research Fellowships“ der Autorin entstanden.

Dr. H. Dumont $(\bowtie)$

Abteilung für Struktur und Steuerung des Bildungswesens, Deutsches Institut für International Pädagogische Forschung (DIPF), Warschauer Str. 34-38, 10243 Berlin, Deutschland

E-Mail: dumont@dipf.de 
concepts used in educational research and findings from research on learning and instruction are presented. Using these insights, the author proposes to conceptualize "Individuelle Förderung" as an overarching teaching concept in which teachers adapt their instruction to each student's needs and aim to increase students' responsibility for their own learning process. The paper closes with implications of this conceptualization for practice and research.

Keywords Adaptive teaching - Individualized instruction · Research on learning and instruction · Teaching

\section{Einleitung}

Das allgemeinbildende Schulwesen ist seit jeher mit der Herausforderung konfrontiert, den unterschiedlichen Bedürfnissen von Schülerinnen und Schülern gerecht zu werden und damit allen Kindern und Jugendlichen eine optimale schulische Entwicklung zu ermöglichen. In Deutschland wurde dies im Sekundarschulbereich bislang vor allem über die Einrichtung von unterschiedlichen Schulformen versucht - mit dem Ziel, homogene Leistungsgruppen zu schaffen. Als Reaktion auf empirische Befunde zur Entstehung von sozialen Ungleichheiten im gegliederten Schulsystem (im Überblick s. Maaz et al. 2009), aber auch aufgrund des Rückgangs der Schülerzahlen gibt es in der jüngeren Vergangenheit zunehmend Bestrebungen zum Zusammenlegen von Schulformen, zur Einrichtung von Gemeinschaftsschulen und zum längeren gemeinsamen Lernen von Schülerinnen und Schülern mit unterschiedlichen Fähigkeitsniveaus. Eine ähnliche Entwicklung ist auch an anderer Stelle des deutschen Schulsystems festzustellen: Seit der UN-Behindertenrechtskonvention, die das Recht von Menschen mit Behinderung auf inklusive Schulbildung formuliert (im Überblick s. Wrase 2015), werden Förderschulen zunehmend abgeschafft und inklusive Schulen eingerichtet. Mit diesen weitreichenden schulstrukturellen Veränderungen geht ein genereller Perspektivwechsel einher, nämlich die Unterschiede zwischen Schülerinnen und Schülern nicht mehr als Problem, sondern als normale Gegebenheit zu betrachten und sie als Ressource für das schulische Lernen zu nutzen (Sliwka 2012; Reusser 2015).

Im Rahmen dieser Diskussion um den adäquaten Umgang mit Heterogenität im Schulkontext wird zunehmend die individuelle Förderung von Schülerinnen und Schülern gefordert (Trautmann und Wischer 2011; Fischer 2014): Individuelle Förderung findet sich in den Schulgesetzen aller Bundesländer wieder, ist ein zentrales Kriterium für Schulentwicklung und Schulevaluation, wird gleichermaßen von Eltern- wie Lehrerverbänden propagiert, ist Gegenstand der Aus- und Weiterbildung von Lehrkräften, gilt als ,notwendiger Steigbügel der Inklusion“ (Fischer 2014, S. 97) und wurde von der Kultusministerkonferenz als „Grundlage für ein Bildungssystem mit Zukunft" bezeichnet (Ahnen 2004). Bei näherer Betrachtung stellt man jedoch fest, dass es kein einheitliches Verständnis des Begriffs und damit sehr unterschiedliche Vorstellungen darüber gibt, wie die individuelle Förderung von Schülerinnen und Schülern erreicht werden soll. Dieser Umstand erschwert nicht nur 
die Umsetzung von individueller Förderung in der Schulpraxis, sondern auch die empirische Untersuchung von individueller Förderung in der Bildungsforschung.

Ziel des vorliegenden Beitrags ist es daher, den in Politik und Praxis populären Begriff der individuellen Förderung näher zu betrachten und vor dem Hintergrund wissenschaftlicher Diskurse für den Unterrichtskontext zu konkretisieren. Dazu wird zunächst auf den Begriff und seine Verwendung eingegangen. Anschließend liegt der Fokus auf der Frage, wie individuelle Förderung im Rahmen des regulären Unterrichts aussehen und umgesetzt werden kann. Hierzu werden im wissenschaftlichen Diskurs etablierte Konzepte zu individueller Förderung sowie empirische Erkenntnisse aus der Unterrichts- und der Lehr-Lern-Forschung herangezogen. Darauf aufbauend wird eine Konzeption von individueller Förderung im Sinne einer Gesamtunterrichtsstrategie vorgeschlagen. Abschließend werden Implikationen für die Praxis und für die empirische Untersuchung von individueller Förderung skizziert.

\section{Zum Begriff ,,individuelle Förderung“" und seiner Verwendung}

Der Begriff ,,individuelle Förderung“ taucht erstmals im Strukturplan des Deutschen Bildungsrats im Jahr 1970 auf. Dort ist die Rede von einem ,,auf individuelle Förderung angelegten Bildungssystem“ (Bildungsrat 1970, S. 27), um ,jeden Lernenden entsprechend seinen Fähigkeiten und Interessen bestmöglich [zu] fördern“ (S. 36). Größere Prominenz hat individuelle Förderung jedoch erst seit der Publikation der Abschlussempfehlungen des von Bund und Ländern getragenen Forums Bildung unmittelbar nach der Veröffentlichung der ersten PISA-Ergebnisse im Jahr 2001 erlangt. Darin heißt es: „Individuelle Förderung entscheidet darüber, ob Menschen sich nach ihren Fähigkeiten und Interessen entwickeln können. Individuelle Förderung ist gleichermaßen Voraussetzung für das Vermeiden und den rechtzeitigen Abbau von Benachteiligungen wie für das Finden und Fördern von Begabungen. [...]. Ziel ist die konsequente Berücksichtigung unterschiedlicher Lernvoraussetzungen [...]. Das erfordert differenzierte Lernangebote, neue Formen des Lehrens und eine zunehmende Selbststeuerung von Lernprozessen durch die Lernenden“ (Forum Bildung 2001, S. 7).

Seitdem hat sich individuelle Förderung, um es mit den Worten von Trautmann und Wischer (2011) auszudrücken, ,zu einer bildungspolitischen Steuerungsstrategie ersten Ranges entwickelt“ (S. 144). Betrachtet man die Verwendung des Begriffs im bildungspolitischen Diskurs jedoch genauer, stellt man schnell fest, dass er selten konkretisiert wird und zudem mit unterschiedlichen Schwerpunktsetzungen einhergeht. Während individuelle Förderung mittlerweile in den Schulgesetzen aller Bundesländer explizit genannt wird, erfolgt in den wenigsten Fällen eine Erklärung, was damit eigentlich gemeint ist. Die Schulgesetze, in denen individuelle Förderung konkretisiert wird, unterscheiden sich zudem darin, wie das Konzept umgesetzt werden soll. Beispielsweise soll laut Thüringer Schulgesetz individuelle Förderung durch binnendifferenzierenden Unterricht, laut Brandenburger Schulgesetz durch die Einrichtung von Leistungs- und Begabungsklassen erfolgen. Darüber hinaus wird der Begriff in den Schulgesetzen häufig in Zusammenhang mit bestimmten Schulformen verwendet, wie zum Beispiel Ganztagsschulen, inklusive Schulen, 
Förderschulen oder Gemeinschaftsschulen. In einigen Bundesländern wird individuelle Förderung hingegen als generelles Lehr- und Lernprinzip aller Schulformen verstanden. Die großen Unterschiede im Verständnis von individueller Förderung zwischen den Schulgesetzen der Bundesländer (s.a. Fischer 2014) lassen sich in ähnlicher Weise auch an anderer Stelle in Bildungspolitik, Bildungsadministration und Bildungspraxis finden. Einig ist man sich allerdings hinsichtlich der Notwendigkeit individueller Förderung zur Erreichung zweier zentraler Ziele: der Verbesserung der Kompetenzen aller Schülerinnen und Schüler sowie der Herstellung von Chancengerechtigkeit (Dräger 2009).

Angesichts der Diskrepanz zwischen den positiven Wirkungserwartungen und der vielfältigen Verwendung und damit einhergehende Unschärfe des Begriffs ,,individuelle Förderung“ ist es nicht überraschend, dass dieser bildungspolitisch geprägte Begriff im wissenschaftlichen Diskurs häufig kritisch gesehen wird. So wird mokiert, dass individuelle Förderung, ,nahezu unhinterfragt als Schlüssel zur Lösung bildungspolitischer und pädagogischer Probleme“ (Klieme und Warwas 2011, S. 805) und als ,eine Art Allheilmittel mit dem Potenzial, jeglichen Missstand und jedes Problem zu kurieren“ (Ebel et al. 2011, S. 1), erscheint. Entsprechend wurde der Begriff bereits als ,,programmatische Nebelbombe“ (Wischer 2012, S. 65) oder „,(politischer) Containerbegriff“ (Maykus et al. 2011, S. 140) bezeichnet. Hasselhorn et al. (2018) merken an, dass der Begriff im wissenschaftslogischen Sinne inhaltsleer ist. Auch wenn der Terminus „Förderung“ ursprünglich aus der Sonderpädagogik stammt und dort für spezifische pädagogische Aufgaben verwendet wurde, können letztlich sämtliche pädagogischen Handlungen mit dem Ziel, einen Menschen zu bilden und zu erziehen, als Förderung verstanden werden (s.a. Ricken 2008). Da Bildungsprozesse immer individuell sind, sei Förderung ,gar nicht anders als individuell zu denken“ (Hasselhorn et al. 2018, S. 4).

Entsprechend werden im wissenschaftlichen Diskurs meist andere Konzepte und Begriffe verwendet, wenn über die Berücksichtigung der individuellen Bedürfnisse von Kindern und Jugendlichen im Schulkontext gesprochen wird (siehe nächstes Kapitel). Unter den Autorinnen und Autoren, die individuelle Förderung explizit thematisieren, gibt es wiederum kein einheitliches Verständnis oder gar eine einheitliche Definition des Begriffs, wie in Tab. 1 erkennbar ist. Für Behrensen und Solzbacher (2012) sowie Eckert (2004) steht beispielsweise die Potenzialentwicklung eines jeden Schülers bzw. einer jeden Schülerin im Vordergrund. Graumann (2008) betont die Notwendigkeit, die gesamte Persönlichkeit eines Kindes in den Blick zu nehmen, und für Kallweit (2015) ist die Verantwortungsübernahme der Schülerinnen und Schüler für ihren eigenen Lernprozess ein zentraler Bestandteil von individueller Förderung. Klieme und Warwas (2011) verstehen unter individueller Förderung ganz allgemein sämtliches ,erzieherisches Handeln unter konsequenter Berücksichtigung personaler Lern- und Bildungsvoraussetzungen“" und unterscheiden drei Varianten eines pädagogischen Verständnisses von individueller Förderung: (1) kompensatorische Trainings- und Zusatzangebote (wie z. B. Nachhilfe), (2) offenen Unterricht und (3) adaptiven Unterricht.

Trotz unterschiedlicher Schwerpunktsetzungen und unabhängig davon, ob sie inner- oder außerschulische Förderung fokussieren, haben die Definitionen gemeinsam, dass sie alle Schülerinnen und Schüler in den Blick nehmen. Das heißt, es 
Tab. 1 Definitionen von individueller Förderung

\begin{tabular}{|c|c|}
\hline Autoren & Definition \\
\hline $\begin{array}{l}\text { Behrensen und Solzba- } \\
\text { cher (2012) } \\
\text { vgl. auch Kunze (2008) }\end{array}$ & $\begin{array}{l}\text { „Unter individueller Förderung in Kita und Grundschule verstehen wir alle } \\
\text { pädagogischen Handlungen, die mit der Intention erfolgen, die Begabungs- } \\
\text { entwicklung und das Lernen jedes einzelnen Kindes zu unterstützen, unter } \\
\text { Aufdeckung und Berücksichtigung seines je spezifischen Potentials, seiner } \\
\text { je spezifischen (Lern-)Voraussetzungen, (Lern-)Bedürfnisse, (Lern-)Wege, } \\
\text { (Lern-)Ziele und (Lern-) Möglichkeiten.“ (S. 5) }\end{array}$ \\
\hline
\end{tabular}

Eckert (2004)

„Individuelles Fördern heißt, jeder Schülerin und jedem Schüler die Chance zu geben, ihr bzw. sein motorisches, intellektuelles, emotionales und soziales Potenzial umfassend zu entwickeln und sie bzw. ihn dabei durch geeignete Maßnahmen zu unterstützen (...).“(S. 97)

Graumann (2008) Einen Schüler individuell zu fördern bedeutet, ihn in seiner gesamten Persönlichkeit ins Auge zu fassen, sein außerschulisches Umfeld in die Förderung einzubeziehen, offen zu sein für seine Interessen und Stärken und, darauf aufbauend, ihn an die Hand zu nehmen, um ihm helfen zu können, seine Schwächen zu beheben." (S. 21-22)

Kallweit (2015) vgl. auch Blaes et al. (2012) „Individuelle Förderung ist das Einrichten von Lernarrangements, die an die diagnostizierten Bedürfnisse jedes einzelnen Schülers adaptiert sind und somit optimale Voraussetzungen und Unterstützung für einen individuellen Lernprozess bieten. Die Schüler werden dabei als aktive Gestalter des eigenen Lernprozesses gesehen, die Verantwortung für ihren Lernerfolg übernehmen." (S. 15)

Klieme und Warwas (2011)

„Zusammenfassend soll hier, individuelle Förderung ‘ als erzieherisches Handeln unter konsequenter Berücksichtigung personaler Lern- und Bildungsvoraussetzungen definiert werden." (S. 808)

Hierbei handelt es sich um alle Definitionen, die zum Zeitpunkt der Literaturrecherche (Mai 2016) zu individueller Förderung gefunden wurden.

geht nicht nur um die Förderung von leistungsschwachen, sondern auch von leistungsstarken Schülerinnen und Schülern, sodass die „Potenzialentfaltung aller Kinder“ ermöglicht wird (Fischer 2014). Dies spiegelt sich auch in den Förderstrategien der Kultusministerkonferenz für leistungsschwache und leistungsstarke Schülerinnen und Schüler, in denen individuelle Förderung gleichermaßen genannt wird (KMK 2010, 2015).

Im vorliegenden Beitrag soll der Fokus darauf liegen, wie individuelle Förderung für alle Schülerinnen und Schüler im regulären Unterrichtsgeschehen aussehen und erreicht werden kann, das heißt, es geht um die ,unterrichtsbezogene Förderung“ (Arnold und Richert 2008) in dem Sinne, dass ,,jede Unterrichtsstunde eine Förderstunde ist und es bei jedem Kind und jedem Jugendlichen Fördermöglichkeiten gibt“" (Höhmann 2009, S. 216). Kompensatorische Trainings- und Zusatzangebote für einzelne Schülerinnen und Schüler sowie außerschulische Fördermaßnahmen werden daher nicht adressiert (s. dazu z. B. Hartmann et al. 2017 bzw. Hardy 2017). 


\section{Der wissenschaftliche Diskurs zu individueller Förderung im Unterricht}

Obwohl der Begriff ,,individuelle Förderung“ im wissenschaftlichen Diskurs eine eher geringe Rolle spielt, hat das Thema eine lange wissenschaftliche Tradition, wie bereits mehrfach angemerkt wurde (Kunze 2008; Trautmann und Wischer 2011; Wischer 2014). Schon zu Beginn des 20. Jahrhunderts wurde die Berücksichtigung der individuellen Lernvoraussetzungen der einzelnen Schülerinnen und Schüler in der Psychologie - als Reaktion auf die Entwicklung psychometrischer Tests zur Erfassung von interindividuellen Unterschieden (Washburne 1925) - und in der Reformpädagogik (im Überblick Graumann 2002) gefordert. Seit den 1970er Jahren wird das Thema sowohl in der Erziehungswissenschaft als auch in der Pädagogischen Psychologie ausführlicher behandelt.

Im Folgenden sollen die zentralen Inhalte dieses wissenschaftlichen Diskurses dargestellt werden. Der Fokus liegt dabei auf der Frage, wie individuelle Förderung im Rahmen des regulären Unterrichts aussehen und umgesetzt werden kann. Da individuelle Förderung nicht allein aus einer Theorie oder einer Forschungsrichtung heraus begründet werden kann (Haag und Streber 2014), wird ein möglichst breiter Zugang gewählt. So werden zunächst eine Reihe von didaktischen Konzepten vorgestellt (Abschn. 3.1), in denen die Berücksichtigung von individuellen Lernvoraussetzungen der Schülerinnen und Schüler im Unterricht thematisiert wird. Wenn vorhanden, werden in diesem Zusammenhang auch empirische Erkenntnisse zur Wirksamkeit dieser Konzepte zusammengefasst. Ergänzend werden anschließend Erkenntnisse aus der kognitionspsychologischen Lehr-Lern-Forschung (Abschn. 3.2) und aus der Unterrichtsforschung (Abschn. 3.3) dargestellt, die Implikationen für individuelle Förderung im Unterricht enthalten.

\subsection{Konzepte zu individueller Förderung im Unterricht}

Im Folgenden wird eine Reihe von Konzepten vorgestellt, die aktuell im wissenschaftlichen Diskurs herangezogen werden, wenn über die Berücksichtigung von individuellen Lernvoraussetzungen der Schülerinnen und Schüler im Unterricht gesprochen wird. Die Konzepte stammen aus unterschiedlichen disziplinären Traditionen und Kontexten und unterscheiden sich somit in vielfältiger Weise, insbesondere hinsichtlich ihrer theoretischen Ursprünge, ihres Praxisbezugs und des Vorliegens empirischer Studien zu ihrer Wirksamkeit. Gleichzeitig gibt es zwischen den Konzepten eine Reihe von Überschneidungen, was nicht selten dazu führt, dass sie von einigen Autorinnen und Autoren synonym verwendet werden. Dies liegt möglicherweise auch darin begründet, dass zu den meisten der vorgestellten Konzepte wiederum unterschiedliche Definitionen und Begriffe existieren, sodass eine trennscharfe Abgrenzung äußerst schwierig ist. Bei der folgenden Darstellung wird jedoch der Versuch unternommen, Unterschiede und Gemeinsamkeiten zwischen den Konzepten herauszuarbeiten. 


\subsubsection{Adaptiver Unterricht}

Das Konzept des adaptiven Unterrichts ist in der Pädagogischen Psychologie zu Hause und basiert auf der theoretischen Annahme der in den 1970er Jahren stark verbreiteten Aptitude-Treatment-Interaktion (ATI)-Forschung (Cronbach und Snow 1977), dass die Effektivität bestimmter instruktionaler Maßnahmen (treatment) von Merkmalen der lernenden Person (aptitude) abhängt. Vor diesem Hintergrund wird mit Begriffen wie adaptive education (Glaser 1972, 1977), adaptive instruction (Cronbach 1967; Cronbach und Snow 1977; Wang und Lindvall 1984) und adaptive teaching (Corno und Snow 1986) im Englischen und adaptiver Unterricht (Schwarzer und Steinhagen 1975; Bönsch 1986; Wember 2001), adaptive Lerngelegenheiten und Adaptivität (Hardy et al. 2011; Hertel 2014) im Deutschen die Anpassung des Unterrichts an die individuellen Lernvoraussetzungen der einzelnen Schülerinnen und Schüler und damit die Schaffung einer möglichst optimalen Lernumwelt für jeden Schüler bzw. jede Schülerin beschrieben. ${ }^{1}$ Die meisten theoretischen und empirischen Arbeiten zum adaptiven Unterricht stammen aus den 1970er und 1980er Jahren. Während diese häufig mehr oder weniger standardisierte ,adaptive educational programs“ - wie beispielsweise das „Adaptive Learning Environments Model“ (ALEM) (Wang 1992) - entwickelten, liegt in neueren Arbeiten der Fokus auf generellen Prinzipien, nach denen adaptiver Unterricht erfolgen kann (s. z. B. Randi und Corno 2005). Die theoretischen Annahmen zum adaptiven Unterricht sind jedoch weitestgehend konstant geblieben, wie die neueste und bislang umfassendste Konzeption von adaptivem Unterricht erkennen lässt, die Corno 2008 vorgelegt hat.

Zentraler Gegenstand des Konzepts von adaptivem Unterricht ist die Bereitstellung eines Unterrichtsangebots, welches zu den individuellen Lernvoraussetzungen der einzelnen Schülerinnen und Schüler passt (Corno 2008). Diese Voraussetzungen ergeben sich nicht nur aus dem Vorwissen und dem aktuellen Leistungsstand, sondern auch aus den Interessen, der Persönlichkeit und einer Vielzahl weiterer lernrelevanter Merkmale der Lernenden (Glaser 1977; Corno und Snow 1986). Dabei kann die Anpassung des Unterrichts auf zwei Ebenen erfolgen (Corno und Snow 1986). Zum einen können Makro-Adaptationen vorgenommen werden. Hiermit sind langfristige und übergeordnete Anpassungen des Unterrichts gemeint, wie die Wahl einer Unterrichtsmethode oder Maßnahmen der Differenzierung, die auf Basis von Leistungsbeurteilungen der Schülerinnen und Schüler durch die Lehrkräfte erfolgen. Zum anderen finden im adaptiven Unterricht kurzfristige, auf informellen Leistungsbeurteilungen basierende Anpassungen des Unterrichts durch die Lehrkraft während eines Tages, einer Unterrichtsstunde und im Rahmen von Lehrer-Schüler-Interaktionen statt. Diese sogenannten Mikro-Adaptationen, die dem Konzept der individuellen Lernunterstützung nach Krammer (2009) und dem contingent support nach Van de Pol et al. (2015) nahestehen, sind der Kern von adaptivem Unterricht (Corno 2008).

\footnotetext{
1 Zwischen den Begriffen adaptive education, adaptive instruction, adaptive teaching bzw. den entsprechenden deutschen Begriffen lassen sich keine konzeptuellen Unterschiede ausmachen. Im vorliegenden Beitrag wird daher der Begriff ,,adaptiver Unterricht“ synonym für diese Begriffe verwendet. Davon abzugrenzen ist jedoch die adaptive Lehrkompetenz, die ein spezifisches Konzept der Arbeitsgruppe um Beck (Beck et al. 2008) darstellt und folgende vier Dimensionen umfasst: Sachkompetenz, diagnostische Kompetenz, didaktische Kompetenz und Klassenführungskompetenz.
} 
Einen weiteren wichtigen Bestandteil der Konzeption von adaptivem Unterricht stellt die Verantwortungsübernahme der Lernenden für ihren eigenen Lernprozess dar. So konstatiert bereits Glaser (1977): ,,[...] an adaptive classroom environment must be designed so that the learner is able to function to an increasing extent as a responsible person participating in decisions about the course of his or her own education“ (S. 80). Je mehr Fähigkeiten der oder die Lernende im Laufe des Unterrichts erwirbt, desto weniger instruktionale Vorgaben sollten durch die Lehrkraft erfolgen, was zunehmend mehr Selbstbestimmung und Selbstständigkeit aufseiten der Lernenden erforderlich macht (Corno und Snow 1986). Somit ist für Corno (2008) adaptiver Unterricht nicht ohne selbstreguliertes Lernen zu denken. Damit die Lehrkräfte wissen, wie viel Unterstützung sie wann bereitstellen müssen bzw. wann sie den einzelnen Schülerinnen und Schülern die Verantwortung übergeben, ist eine kontinuierliche, informelle Evaluation des Lernfortschritts erforderlich (Randi und Corno 2005; Corno 2008). Schließlich wird in Arbeiten zum adaptiven Unterricht die Notwendigkeit angesprochen, den Unterricht anders zu organisieren als in festen Gruppierungen von Schülerinnen und Schülern, die das Curriculum im Gleichschritt durchlaufen (Glaser 1977; Corno 2008). Dies bedeutet nicht, dass die Lernenden ausschließlich für sich allein arbeiten, sondern dass instruktionsbasiertes und kooperatives Lernen ebenso Bestandteil eines adaptiven Unterrichts sind (Waxman et al. 1985; Wang und Zollers 1990) und Lernen weiterhin innerhalb eines Gruppenkontextes stattfindet (Corno 2008).

Trotz der umfangreichen theoretischen Auseinandersetzung mit adaptivem Unterricht und Beispielen in der Literatur, wie dies in der Praxis aussehen kann (siehe z. B. Randi und Corno 2005; Parsons 2012; Allen et al. 2013), gibt es keine empirische Evidenz zum neuesten Konzept des adaptiven Unterrichts nach Corno (2008). Die empirischen Arbeiten zum Thema stammen aus den 1970er und 1980er Jahren und fokussierten auf die Evaluation der entwickelten ,adaptive educational programs“. Diesbezüglich kommen Waxman et al. (1985) in ihrer Forschungssynthese zu dem Schluss, dass solche Programme einen substanziellen Effekt auf Kompetenzen von Schülerinnen und Schülern haben. Da die Autorinnen und Autoren jedoch kaum Informationen über die herangezogenen 38 Einzelstudien zur Verfügung stellen, ist die methodische Qualität der Studien vor dem Hintergrund heutiger Standards schwer zu beurteilen. Die aus der gleichen Zeit stammenden Arbeiten zu spezifischen Aptitude-Treatment-Interaktionen geben jedoch einige Hinweise für die Wirksamkeit von adaptivem Unterricht. So konnte mehrfach gezeigt werden, dass leistungsschwache Schülerinnen und Schüler von einer hohen Lehrersteuerung und Strukturierung des Unterrichts profitieren, während sich leistungsstarke Schülerinnen und Schüler in einem stärker selbstgesteuerten und entdeckenden Unterrichtssetting entwickeln (Cronbach und Snow 1977; Snow 1989). Dieser Befund konnte auch in neueren Studien mehrfach bestätigt werden (siehe z. B. Connor et al. 2004; Decristan et al. 2015a; s.a. Kalyuga 2007, für einen Überblick zu Experimentalstudien zu ATIEffekten). 


\subsubsection{Binnendifferenzierung}

In Abgrenzung zur äußeren Differenzierung - die Einteilung von Schülerinnen und Schülern in feste Gruppen auf Basis bestimmter Kriterien - wird unter Binnendifferenzierung bzw. dem synonym verwendeten Begriff innerer Differenzierung eine gruppeninterne Differenzierung verstanden, in der die Lerninhalte gezielt für verschiedene, flexibel eingesetzte kleinere Schülergruppen variiert werden. Die Gruppen werden dabei auf der Basis bestimmter Merkmale der Schülerinnen und Schüler, wie etwa ihrem Kompetenzstand, zusammengesetzt (Klafki und Stöcker 1976; Bönsch 2004). Es handelt sich hierbei um ein aus der Allgemeinen Pädagogik und Schulpädagogik stammendes didaktisches Konzept, das ähnlich wie das Konzept des adaptiven Unterrichts in den 1970er Jahren stark diskutiert und propagiert wurde und heute noch aktuell ist (Trautmann und Wischer 2008). Der Schwerpunkt liegt auf der konkreten, praktischen Umsetzung im Unterricht - ein Aspekt, der in den Arbeiten zum adaptiven Unterricht nur am Rande auftaucht. Im Englischen ist das Konzept unter differentiated instruction (siehe z. B. McTighe und Brown 2005; Constas und Sternberg 2006) zu finden, aber auch die Konzepte within-class grouping (siehe z.B. Lou et al. 1996) und ability grouping (siehe z. B. Slavin 1987) bezeichnen im Englischen das Gruppieren von Schülerinnen und Schülern innerhalb von Klassen mit dem Ziel, differenzierte Unterrichtsangebote machen zu können.

In Anlehnung an Bräu und Schwerdt (2005) sowie Brüning und Saum (2010) lassen sich fünf Formen der Differenzierung unterscheiden, die jedoch eng miteinander zusammenhängen und häufig gemeinsam auftreten: methodische (Variation der Aufgabenstellungen und Aufgabenformate), mediale (Variation der Arbeitsmaterialien), quantitative (Variation der Bearbeitungszeit und des Aufgabenumfangs), qualitative (Variation der Lernziele und des Schwierigkeitsniveaus) und inhaltliche Differenzierung (Variation der Aufgabeninhalte und Themen). Den „Dreh- und Angelpunkt zur Planung, Vorbereitung, Zusammenführung und Auswertung unterschiedlicher Zugangs-, Lösungs- und Erkenntniswege“ (S. 21) bildet der Klassenunterricht bzw. der Klassenverband (Scholz 2007).

Wenngleich das Konzept der Binnendifferenzierung zum Ziel hat, den Unterricht an die Bedürfnisse und Lernvoraussetzungen der Schülerinnen und Schüler anzupassen (Paradies und Linser 2008), wurde darauf hingewiesen, dass die Variation von Lerninhalten und weiteren Unterrichtsaspekten nicht automatisch adaptiv ist, also dem jeweiligen Entwicklungsstand der Schülerinnen und Schüler angemessen (Wischer 2007; Hertel 2014). Weiterhin ist das Unterrichtsangebot im binnendifferenzierten Unterricht adaptiv für die zusammengestellten Schülergruppen, nicht jedoch für einzelne Schülerinnen und Schüler (Arnold und Richert 2008). Somit findet sich hier ein entscheidender Unterschied zum Konzept des adaptiven Unterrichts. Darüber hinaus ist das selbstregulierte Lernen der Schülerinnen und Schüler kein integraler Bestandteil des Konzepts der Binnendifferenzierung, wie dies beim Konzept des adaptiven Unterrichts der Fall ist. Die von Heymann (2010) eingeführte Unterscheidung in geschlossene Binnendifferenzierung, in welcher die Lehrkraft den Lernenden unterschiedliche Lernwege zuweist, und offene Binnendifferenzierung, in welcher die Lernenden ihre individuellen Lernwege selbst finden sollen, rückt das Konzept jedoch in die Nähe von adaptivem Unterricht. 
Hinsichtlich der Wirksamkeit von Binnendifferenzierung wurden die meisten Studien im angloamerikanischen Raum im Kontext des ability groupings durchgeführt. Sie kommen übereinstimmend zu dem Ergebnis, dass das Gruppieren von Schülerinnen und Schülern innerhalb von Klassen per se keine oder nur sehr geringe Effekte auf deren Kompetenzentwicklung hat (Kulik und Kulik 1982; Slavin 1987, 1990; Lou et al. 1996; Mulkey et al. 2005; Schofield 2010). Wenn die Gruppierungsmaßnahmen mit einem differenzierten Unterrichtsangebot und einer kontinuierlichen Überprüfung der Kompetenzen der Schülerinnen und Schüler einhergeht, finden sich etwas größere Effekte (Slavin 1987; Gutierrez und Slavin 1992; Lou et al. 1996). Hattie (2009) kommt in seiner Meta-Metaanalyse zu dem Schluss, dass Schülerinnen und Schüler in einem Unterricht mit binnendifferenzierten Angeboten kaum mehr dazu lernen als Schülerinnen und Schüler in einem Unterricht ohne solche Maßnahmen.

\subsubsection{Individualisierung}

Im Gegensatz zum adaptiven Unterricht und zur Binnendifferenzierung ist Individualisierung (oder auch synonym: individualisiertes Lernen und individualisierter Unterricht) ein jüngerer Begriff, der erst in den letzten zehn Jahren in der Literatur, vor allem im Bereich der Schulpädagogik, auftaucht (siehe z. B. Bräu 2005; Czerwanski 2006; Wellenreuther 2008; Paradies et al. 2010; Bohl et al. 2011; Breidenstein 2014; Rabenstein und Wischer 2016). Es handelt sich hier nicht um ein klar umgrenztes Konzept; der Begriff wird je nach Autor oder Autorin sehr unterschiedlich verstanden. Es lassen sich jedoch grob zwei unterschiedliche Sichtweisen unterscheiden (Schneuwly 2014). So gibt es einerseits eine Gruppe von Autorinnen und Autoren, die Individualisierung als Extremform von Binnendifferenzierung verstehen, in der die Differenzierung nicht für Schülergruppen, sondern für jeden einzelnen Schüler und jede einzelne Schülerin erfolgt (Bönsch 2004; Bohl et al. 2011). Andererseits wird Individualisierung als eine Art Oberbegriff verwendet, um einen dezentrierten Unterricht zu beschreiben, in dem die Schülerinnen und Schüler zur gleichen Zeit Unterschiedliches tun und Kommunikations- und Interaktionsprozesse zwischen Lehrkraft und allen Schülerinnen und Schülern in den Hintergrund treten (Wellenreuther 2008; Breidenstein 2014; Lipowsky und Lotz 2015; Breidenstein und Rademacher 2016). Auch im Englischen steht individualized instruction trotz der langen Tradition des Begriffs (Courtis 1938) - nicht für ein klar definiertes Konzept, sondern wird meistens als Sammelbegriff verwendet: „Individualized instruction refers to the idea that each student learns differently and thus in order to accommodate these differences, instruction should be personalized, matched, or adapted to the experiences, attitudes, and interests of each individual student" (Waxman et al. 2013).

Da unter Individualisierung sehr Unterschiedliches verstanden wird, ist eine Beurteilung der Wirksamkeit nicht einfach. Dennoch geben ältere angloamerikanische Studien Hinweise darauf, dass individualized instruction nur geringe Effekte auf die Leistungen von Schülerinnen und Schülern hat (Horak 1981; Bangert et al. 1983). Dies wird auch durch die Meta-Metaanalyse von Hattie (2009) bestätigt. Hess und Lipowsky (2017) führen die geringe Effektivität von individualisiertem Unterricht 
darauf zurück, dass das fachliche Niveau von Schüler-Lehrer-Interaktionen in solch einem Unterricht eher gering ausgeprägt ist. In der Tat lassen sich für diese Annahme empirische Belege aus jüngerer Zeit finden. So beobachten sowohl Krammer (2009) als auch Lotz (2015) in ihren Videostudien, dass in Schülerarbeitsphasen kaum kognitiv aktivierende Interaktionen zwischen Lehrkräften und Schülerinnen und Schülern stattfinden. Auch Breidenstein und Rademacher (2017) berichten auf Basis ihrer teilnehmenden Beobachtung an vier Schulen, in denen individualisiert unterrichtet wird, von einer äußerst geringen inhaltlichen Auseinandersetzung mit dem Unterrichtsgegenstand. Möglicherweise führt die Dezentralisierung des Unterrichts zu einer solchen Komplexität für die Lehrkraft, dass die Orchestrierung der verschiedenen Aktivitäten der Schülerinnen und Schüler und weniger der inhaltliche Austausch im Mittelpunkt steht (Lipowsky und Lotz 215). Dass Individualisierung aber auch erfolgreich umgesetzt werden kann, zeigt eine Interventionsstudie von Connor et al. (2009), in der Unterricht mit individualisierten, computergestützten Aufgaben, die kontinuierlich an das Leistungsniveau der Schülerinnen und Schüler angepasst wurden, zu einer besseren Leseentwicklung führte als Unterricht ohne individualisierte Aufgaben.

\subsubsection{Personalisiertes Lernen}

Obwohl es sich beim personalisierten Lernen nicht um ein klar definiertes Konzept, sondern vielmehr um einen bildungspolitischen Begriff handelt (Johnson 2004), soll es der Vollständigkeit halber an dieser Stelle genannt werden. Im deutschsprachigen Raum wird der Begriff kaum verwendet (mit Ausnahme der Schweiz, wo er ähnlich wie Individualisierung in Deutschland verwendet wird, siehe z. B. das Projekt ,perLen - personalisierte Lernkonzepte in heterogenen Lerngruppen“, Reusser 2015); vielmehr ist er im englischsprachigen Raum unter personalised learning oder personalisation zu finden (Diack 2004; Johnson 2004; Burton 2007; Campbell et al. 2007; Clarke 2013). Je nach Autor oder Autorin wird personalisiertes Lernen sehr unterschiedlich verstanden. Während Diack (2004) und Campbell et al. (2007) hierunter ganz allgemein ,tailored education“ verstehen, betont beispielsweise Clarke (2013), dass von personalisiertem Lernen nur dann gesprochen werden sollte, wenn die Schülerinnen und Schüler Kontrolle und Verantwortung für ihren eigenen Lernprozess übernehmen. Zusammenfassend lässt sich feststellen, dass der Begriff im wissenschaftlichen Diskurs eher eine untergeordnete Rolle spielt und entsprechend keine empirischen Studien vorliegen.

\subsubsection{Offener Unterricht}

Beim offenen Unterricht handelt es sich um ein reformpädagogisch geprägtes Konzept, das seit den 1970er Jahren vor allem in der schulpädagogischen Literatur diskutiert wird und durch die Selbst- und Mitbestimmungsmöglichkeiten der Schülerinnen und Schüler im Unterricht gekennzeichnet ist (Bohl und Kucharz 2010). Nach Peschel (2002) kann eine Öffnung des Unterrichts in folgenden vier Dimensionen stattfinden: durch organisatorische Öffnung (Flexibilität in Bezug auf Raum, Zeit und Sozialform), methodische Öffnung (verschiedene Lernmethoden stehen 
zur Wahl), inhaltliche Öffnung (die Lernenden bestimmen ihre Lerninhalte selbst) und soziale bzw. politisch-partizipative Öffnung (demokratische Mitbestimmung der Lernenden beim Unterrichtsablauf und bei Unterrichtsregeln). In Anlehnung an diese Unterscheidung schlagen Bohl und Kucharz (2010) vor, nur dann von offenem Unterricht zu sprechen, wenn eine Mitbestimmung der Schülerinnen und Schüler in inhaltlicher und/oder politisch-partizipativer Hinsicht vorliegt. Eine Beteiligung in organisatorischer und methodischer Hinsicht sollte hingegen lediglich als Öffnung des Unterrichts bezeichnet werden. Ähnlich wie beim Konzept der Binnendifferenzierung liegt der Schwerpunkt in der Literatur zum offenen Unterricht bzw. zur Öffnung von Unterricht auf der konkreten Umsetzung im Unterricht. Es handelt sich darüber hinaus um ein im deutschsprachigen Raum verbreitetes Konzept, das nicht mit den englischsprachigen Konzepten open learning und open education gleichzusetzen ist, die sich eher auf eine institutionelle Öffnung von Lernprozessen beziehen (siehe z.B. Lewis 1986). Es ist jedoch dem englischsprachigen Konzept des discovery learning sehr ähnlich, bei dem sich die Schülerinnen und Schüler mit den Unterrichtsinhalten aktiv und weitestgehend eigenständig auseinandersetzen sollen (Mayer 2004).

Die meisten empirischen Befunde zur Wirksamkeit von geöffnetem Unterricht stammen aus dem angloamerikanischen Kontext und kommen mit großer Übereinstimmung zu dem Schluss, dass offener, entdeckender Unterricht mit geringer Steuerung durch die Lehrkraft weniger effektiv ist als lehrergelenkter Unterricht (Mayer 2004; Alfieri et al. 2011) und sogar negative Auswirkungen auf den Lernprozess von Schülerinnen und Schülern haben kann (Kirschner et al. 2006). Wenn die Öffnung des Unterrichts hingegen mit einer Steuerung und kognitiven Strukturierung durch die Lehrkraft einhergeht, ist diese Unterrichtsform dem lehrergelenkten Unterricht überlegen (Hardy et al. 2006; Alfieri et al. 2011). Wichtig ist, dass die Schülerinnen und Schüler ausreichende Kompetenzen und das notwendige Vorwissen haben, um sich eigenständig mit dem Unterrichtsgegenstand auseinandersetzen zu können (Lipowsky und Lotz 2015). Daher brauchen sie je nach Fähigkeitsniveau einen unterschiedlichen Grad an Strukturierung durch die Lehrkraft (siehe ATI-Effekte).

\subsubsection{Formative Assessment}

Hinter dem Begriff formative assessment verbirgt sich ein Konzept, zu dem im englischsprachigen Raum seit 30 Jahren eine Vielzahl von theoretischen und empirischen Arbeiten veröffentlicht worden sind (Sadler 1989; Black und Wiliam 1998, 2009; Andrade und Cizek 2009; Kingston und Nash 2011) und das darüber hinaus in vielen Ländern Einzug in Bildungspolitik und Bildungspraxis gefunden hat (Looney 2005). Im Gegensatz zum summative assessment, welches im Unterrichtskontext die abschließende Leistungsbewertung der Schülerinnen und Schüler am Ende einer Lerneinheit darstellt, beschreibt formative assessment eine kontinuierliche und den Lernprozess begleitende Beurteilung und Rückmeldung mit dem Ziel, den Unterricht zu verbessern und ihn besser an die Bedürfnisse der Schülerinnen und Schüler anzupassen (Sadler 1989; Wiliam 2010). Formative assessment stellt also keine singuläre Unterrichtsmethode dar, impliziert jedoch ein umfassendes Unterrichtskonzept, in dem die Lernvoraussetzungen der Schülerinnen und Schüler berücksichtigt werden. 
Vor diesem Hintergrund wird das Konzept von einigen Autoren auch assessment for learning genannt (s. im Überblick Wiliam 2010) und kann als ,the bridge between teaching and learning" (Wiliam 2010, S. 137) verstanden werden.

Die inhaltliche Nähe zum Konzept des adaptiven Unterrichts ist unmittelbar offensichtlich: Die für die Mikro-Adaptationen notwendigen informellen Leistungsbeurteilungen stellen letztlich nichts anderes als formative assessment dar (Decristan et al. 2017). Dies wird auch bei Wiliam (2010) deutlich, wenn er schreibt, dass formative assessment dazu da ist, ,,moments of contingency“ herzustellen und ,differential treatments“ der Lehrkraft notwendig macht. Auch weist formative assessment eine Nähe zu Konzepten des selbstregulierten Lernens und dem Gedanken der Selbstbestimmung im offenen Unterricht auf. So wird in der Literatur zum formative assessment explizit betont, dass die Beurteilung nicht nur durch die Lehrkraft und teilweise durch die Mitschülerinnen und Mitschüler, sondern auch durch die Lernenden selbst erfolgen sollte (Sadler 1989; Black und Wiliam 2009; s. a. Butler und Winne 1995, zum Zusammenhang zwischen Feedback und selbstreguliertem Lernen). Die Wirksamkeit von formative assessment bzw. einer lernbegleitenden Diagnostik in Verbindung mit konstruktivem Feedback wurde inzwischen in vielen Studien gezeigt (Black und Wiliam 1998; Hattie 2009; Kingston und Nash 2011; Decristan et al. 2015b).

\subsubsection{Selbstreguliertes Lernen}

Beim selbstregulierten Lernen (englisch: self-regulated learning; teilweise synonym verwendet: selbstgesteuertes, selbstorganisiertes oder selbstbestimmtes Lernen) handelt es sich im Gegensatz zu den bisher dargestellten Konzepten nicht um einen Unterrichtsansatz, sondern um ein psychologisches Konzept zur Beschreibung der Fähigkeiten von Schülerinnen und Schülern, ihren eigenen Lernprozess zu initiieren, zu organisieren und zu überwachen (Otto et al. 2015). Da diese Fähigkeit jedoch als wichtiges Ziel von individueller Förderung angesehen werden kann (Klieme und Warwas 2011) und in fast allen der vorgestellten Unterrichtskonzepte eine entscheidende Rolle spielt, soll das Konzept an dieser Stelle erläutert werden.

Das Konzept des selbstregulierten Lernens stammt aus der Pädagogischen Psychologie und ist hier seit den 1980er Jahren sowohl international als auch national ein prominentes Forschungsthema (Zimmerman und Schunk 1989; Zimmerman 1990; Boekaerts 1999; Boekaerts et al. 2000; Schmitz 2001; Winne 2005; Wirth und Leutner 2008). Ähnlich wie bei den anderen bislang vorgestellten Konzepten existieren auch für selbstreguliertes Lernen eine Vielzahl unterschiedlicher Definitionen und Konzeptionen. Nach Pintrich (2000) lässt sich selbstreguliertes Lernen zusammenfassend definieren als ,ein aktiver, konstruktiver Prozess, bei dem der Lernende sich Ziele für sein Lernen selbst setzt und zudem seine Kognitionen, seine Motivation und sein Verhalten in Abhängigkeit von diesen Zielen und den gegebenen äußeren Umständen beobachtet, reguliert und kontrolliert" (S. 453; Übersetzung von Otto et al. 2015, S. 42). Selbstreguliertes Lernen ist demnach ein umfassendes Konzept, welches Aussagen über das Zusammenspiel von kognitiven, metakognitiven und motivationalen Fähigkeiten von Lernenden macht. Ein entscheidender Grundgedanke dabei ist, dass kognitive und motivationale Prozesse beim Lernen wechselseitig von- 
einander abhängig sind und nicht getrennt voneinander verstanden werden können (Zimmerman 1990). Die zum selbstregulierten Lernen entwickelten Modelle lassen sich grob in zwei Gruppen einteilen (Otto et al. 2015; nach Winne und Perry 2000): Während in sogenannten Komponentenmodellen verschiedene Teilkompetenzen des selbstregulierten Lernens beschrieben werden (siehe z. B. Boekaerts 1999), wird in Prozessmodellen der Prozess des selbstregulierten Lernens anhand von verschiedenen Phasen dargestellt (siehe z. B. Zimmerman 2000).

Aus den empirischen und theoretischen Arbeiten zum selbstregulierten Lernen lassen sich auch Implikationen für den Unterricht ableiten. So gibt es inzwischen eine Reihe von (Interventions-)Studien dazu, wie selbstreguliertes Lernen im Unterricht gefördert werden kann (Paris und Paris 2001). Dabei unterscheiden sich jedoch die Annahmen darüber, wie dies geschehen sollte (im Überblick s. Paris und Paris 2001; Boekaerts und Corno 2005). Zum einen gibt es den Ansatz, dass Selbstregulationsstrategien (z.B. Zielsetzung, Selbstmotivation) von der Lehrkraft zusätzlich zu den Fachinhalten als übergeordnete Kompetenzen direkt trainiert werden. Ein anderer Ansatz betont, dass selbstreguliertes Lernen im Laufe des Aufbaus von Fachkompetenzen mitentwickelt wird, indem die Lernenden je nach Kompetenzstand kontinuierlich mehr Verantwortung für ihren eigenen Lernprozess übertragen bekommen. Während der erste Ansatz nicht direkt im Zusammenhang mit den bereits dargestellten Unterrichtskonzepten steht und auch im Regelunterricht umgesetzt werden kann, entspricht der zweite Ansatz unmittelbar dem Konzept des adaptiven Unterrichts (Corno 2008). Vor diesem Hintergrund kann mit den Worten von Zimmerman (1990) konstatiert werden: ,A self-regulated learning perspective [...] has profound implications for the way teachers should interact with students and the manner in which schools should be organized" (S. 4).

\subsection{Erkenntnisse aus der kognitionspsychologischen Lehr-Lern-Forschung}

Während die im vorangehenden Abschnitt dargestellten Konzepte den Lernprozess von Schülerinnen und Schülern im realen Unterrichtskontext in den Blick nehmen, gibt es ein grundlagenwissenschaftliches Forschungsfeld, das sich primär auf der Basis von experimentellen Laborstudien mit der Frage beschäftigt, wie Menschen lernen und wie Lernumgebungen gestaltet werden können, um Lernprozesse zu optimieren (im Überblick s. Bransford et al. 2000; Dumont et al. 2010; Schnotz 2016). Die Ursprünge und theoretischen Fundamente dieses Forschungsfeldes liegen in der Lern- und Kognitionspsychologie. Inzwischen ist jedoch eine Vielzahl weiterer Disziplinen (z. B. die Neurowissenschaften) beteiligt, sodass sich im Englischen auch der Begriff learning sciences etabliert hat (Sawyer 2006).

Im Rahmen dieser Forschung ist die sozialkonstruktivistische Lerntheorie die aktuell vorherrschende Theorie des Wissenserwerbs (Bransford et al. 2000; De Corte 2010; Schneider und Stern 2010; Schnotz 2016). Danach wird das Erlernen von Wissen und Kompetenzen als aktiver, idiosynkratischer Prozess verstanden, bei dem kognitive Strukturen aufgebaut werden. Dabei werden neue Informationen mit bereits existierenden Strukturen, das heißt mit dem jeweiligen Vorwissen, im Sinne eines hierarchischen Systems verbunden. Dieser Prozess findet nicht isoliert, sondern immer in Interaktion des Lernenden mit seiner sozialen und kulturellen Umgebung 
statt und ist somit in einen situativen Kontext eingebettet. Entscheidend für die Qualität des aufgebauten Wissens, also die Verständnistiefe eines Sachverhalts, ist, dass neues Wissen nicht einfach nur kumuliert wird, sondern dass die Wissensstrukturen von Lernenden kontinuierlich ausgebaut, differenziert, integriert, systematisiert und organisiert werden. Entsprechend der cognitive load theory (Sweller et al. 1998; Sweller 2003) muss hierbei jedoch berücksichtigt werden, dass die kognitive Verarbeitungskapazität des menschlichen Gehirns begrenzt ist und somit eine kognitive Überlastung durch zu viele oder zu schwierige Informationen dazu führt, dass neues Wissen gar nicht oder nur auf oberflächliche Art gespeichert wird. Aber auch zu wenig kognitive Aktivierung hemmt den Lernprozess, das heißt, wenn der Lernende nicht dazu herausgefordert wird, bestehende Wissensstrukturen weiterzuentwickeln,.

Die kognitive Verarbeitungskapazität bestimmt sich ganz individuell für jeden Lernenden über das bereits vorhandene Vorwissen in einer Wissensdomäne sowie über seine kognitiven Grundfähigkeiten. Aufgabe der Lehrkraft ist es demnach, den Bereich zwischen Unterforderung und Überforderung für die einzelnen Lernenden zu finden und das Lernangebot daraufhin anzupassen (Van Merriënboer und Sweller 2005). In diesem Zusammenhang spricht Vygotsky (1963) von Zonen der proximalen Entwicklung. Er meint damit den Entwicklungsbereich zwischen dem aktuellen Entwicklungsstand eines Lernenden und seinem potenziellen Entwicklungsstand, den er mit externer Unterstützung erreichen kann. Die Lehrkraft begleitet die Lernenden also durch diese Entwicklungszonen, wobei die von ihr bereitgestellte externe Unterstützung auch als Scaffolding bekannt ist (Wood et al. 1976). Van de Pol et al. (2010) verstehen Scaffolding als interaktiven Prozess zwischen Lehrkraft und Lernendem, der folgendermaßen gekennzeichnet ist: (1) Die Lehrkraft stellt eine adaptive Unterstützung bereit, (2) die Unterstützung durch die Lehrkraft wird zurückgenommen und (3) die Verantwortung für den Lernprozess langsam auf den Lernenden übertragen. Die zentrale Herausforderung besteht für Lehrkräfte demnach darin, für jeden Lernenden die richtige Menge und Art von Unterstützung bereitzustellen und damit für den optimalen Grad an kognitiver Aktivierung zu sorgen.

\subsection{Erkenntnisse aus der Forschung zu Unterrichtsqualität}

Auch wenn die empirische Forschung zu Unterrichtsqualität fast ausschließlich auf Beobachtungen von Unterrichtssettings basiert, in denen alle Schülerinnen und Schüler das gleiche Unterrichtsangebot durch die Lehrkraft erhalten, liefert sie wertvolle Erkenntnisse für die Umsetzung von individueller Förderung im Unterricht. Hier ist insbesondere die konzeptuelle Unterscheidung zwischen sogenannten Sicht- und Tiefenstrukturen des Unterrichts zu nennen (Helmke 2009; Kunter und Trautwein 2013). Während es sich bei den Sichtstrukturen um beobachtbare Merkmale des Unterrichts, wie Organisationsformen, Methoden oder Sozialformen, handelt, beschreiben die Tiefenstrukturen die Interaktionen zwischen Lehrenden und Lernenden und die Art und Weise der Lernstoffvermittlung. Dabei wird davon ausgegangen, dass sich die Qualität und damit die Wirksamkeit von Unterricht nicht aus den Sichtstrukturen ergeben, sondern nur über die Tiefenstrukturen erfasst werden kann. Hess und Lipowsky (2017) merken in diesem Zusammenhang an, dass Maßnahmen der Individualisierung und der Öffnung von Unterricht häufig die methodische Organisation 
des Unterrichts und damit die Sichtstruktur von Unterricht betreffen, jedoch nicht notwendigerweise mit einer Veränderung oder Verbesserung der Tiefenstrukturen einhergehen.

Zur Beschreibung der Tiefenstrukturen des Unterrichts und damit der Unterrichtsqualität haben sich in der deutschsprachigen Unterrichtsforschung in den vergangenen Jahren drei „Basisdimensionen“ guten Unterrichts etabliert: Klassenführung, Unterstützung und kognitive Aktivierung (Klieme 2006; Klieme und Rakoczy 2008; Kunter und Trautwein 2013). Bei Klassenführung geht es um die Steuerung und Strukturierung des Unterrichts durch die Lehrkraft, sodass die Unterrichtszeit effektiv genutzt werden kann. Mit der Dimension Unterstützung ist die Schaffung eines schülerorientierten und verständnisvollen Lernklimas gemeint. Kognitive Aktivierung bezieht sich darauf, inwiefern die Lernenden durch den Unterricht dazu angeregt werden, sich vertiefend mit dem Lernstoff auseinanderzusetzen, und knüpft damit direkt an die Erkenntnisse aus der kognitionspsychologischen Lehr-Lern-Forschung an. Diese drei Dimensionen weisen wiederum große Ähnlichkeit mit den Kategorien des international viel genutzten Instruments CLASS zur Unterrichtsbeobachtung auf: organisational, emotional und instructional support (Pianta und Hamre 2009).

\section{Individuelle Förderung als Gesamtunterrichtsstrategie: Vorschlag einer Konzeption}

Betrachtet man die im vorangegangenen Abschnitt dargestellten Erkenntnisse des wissenschaftlichen Diskurses, wird deutlich, dass in einem Unterricht, in dem die Lehrkraft das gleiche Lernangebot für alle Schülerinnen und Schüler bereitstellt, viele Lernende, insbesondere die am unteren und oberen Ende des Leistungsspektrums, nicht optimal gefördert werden (Trautmann und Wischer 2011). Insofern steht die Forderung nach einem Unterricht, in dem die Bedürfnisse der einzelnen Schülerinnen und Schüler berücksichtigt werden, im Einklang mit den Erkenntnissen der Bildungs- bzw. Lehr-Lern- und Unterrichtsforschung (Bransford et al. 2000; Diesbergen 2010; Hardy et al. 2011; Wischer 2012). Wie lässt sich nun aber individuelle Förderung im Rahmen des regulären Unterrichts, das heißt im Gruppen- und Klassenkontext, umsetzen?

Im Folgenden soll der Versuch unternommen werden, die aus verschiedenen Forschungstraditionen stammenden Konzepte und empirischen Erkenntnisse zu einer disziplinübergreifenden Konzeption von individueller Förderung im Unterricht zu verbinden. Dabei wird das im öffentlichen Diskurs der individuellen Förderung zugrunde liegende „Leitmotiv der Potenzialentfaltung“ (Fischer 2014) aller Schülerinnen und Schüler als Ausgangbasis genommen. Des Weiteren wird die Position vertreten, dass individuelle Förderung im Unterricht eine Gesamtunterrichtsstrategie darstellen muss, die sich nicht auf einzelne Fördermaßnahmen oder spezifische Unterrichtsmethoden beschränkt (wenngleich kompensatorische Förderangebote selbstverständlich Teil solch einer Gesamtunterrichtsstrategie sein können), sondern sämtliche Unterrichtsaktivitäten einschließt und die Tiefenstrukturen des Unterrichts adressiert. 
Für solch eine Gesamtunterrichtsstrategie eignet sich das Konzept des adaptiven Unterrichts als Grundgerüst, da es das umfassendste und nach wie vor ,,wissenschaftlich fundierteste und didaktisch aussichtsreichste unterrichtliche Konzept [ist], um auf die großen [...] interindividuellen Unterschiede der Schüler in didaktisch angemessener Form zu reagieren“ (Helmke und Weinert 1997, S. 137). Das heißt, in einem individuell fördernden Unterricht wird das Unterrichtsangebot kontinuierlich an die individuellen Lernvoraussetzungen der einzelnen Schülerinnen und Schüler angepasst (siehe auch Hardy 2017). Zu diesen individuellen Lernvoraussetzungen zählen insbesondere die kognitiven Grundfähigkeiten sowie das Vorwissen der Schülerinnen und Schüler hinsichtlich des jeweiligen Unterrichtsgegenstandes. Aber auch emotionale, motivationale und volitionale Merkmale sind entscheidend dafür, welches Unterrichtsangebot die einzelnen Lernenden brauchen. Letztlich beeinflussen alle Merkmale einer Person, das heißt auch ihr Geschlecht, ihr Alter, ihr familiärer, kultureller und sprachlicher Hintergrund sowie ihre Persönlichkeit, den Lernprozess und sollten daher von der Lehrkraft berücksichtigt werden (Glaser 1977; Corno und Snow 1986; Randi und Corno 2005; Hertel et al. 2016). Genauso wie sich die Lernvoraussetzungen der Schülerinnen und Schüler durch den Unterricht entwickeln und verändern, muss auch das Unterrichtsangebot kontinuierlich modifiziert werden (Corno 2008). Dabei gilt es insbesondere das Spannungsfeld zwischen individuellem und gemeinschaftlichem Lernen auszuloten bzw. eine entsprechende Balance zu finden, indem zum Beispiel verschiedene Lösungswege einer Aufgabe gemeinsam diskutiert oder Klassenkameradinnen und -kameraden in den individuellen Lernprozess einzelner Schülerinnen und Schüler einbezogen werden (Hardy 2017).

Um das Unterrichtsangebot für die einzelnen Schülerinnen und Schüler kontinuierlich anpassen zu können, ist es notwendig, ihre Kompetenzen im Sinne des formative assessments regelmäßig in formeller und informeller Weise zu evaluieren. Dies erfordert von der Lehrkraft sowohl eine hohe diagnostische Kompetenz als auch ein großes fachliches und fachdidaktisches Wissen des zu vermittelnden Sachverhalts (Behrensen et al. 2015). Gleichzeitig sollten die Schülerinnen und Schüler im Laufe der Zeit zunehmend dazu befähigt werden, ihre eigenen Kompetenzen einschätzen und ihren eigenen Lernprozess steuern zu können (Corno 2008; Van de Pol et al. 2010), wobei für jeden Einzelnen die richtige Balance zwischen Steuerung durch die Lehrkraft und Selbststeuerung gefunden werden muss (De Corte 2010). Die Förderung selbstregulativer Fähigkeiten im adaptiven Unterricht ist nicht nur für sich genommen wünschenswert, sondern auch eine zentrale Voraussetzung dafür, dass die Lehrkraft im Unterricht Zeitfenster schaffen kann, um mit einzelnen Schülerinnen und Schülern intensiver zu interagieren (Helmke 2013).

Die Anpassung des Unterrichtsangebots an die Lernvoraussetzungen der Schülerinnen und Schüler lässt sich durch den Einsatz von Methoden der Binnendifferenzierung in quantitativer, methodischer, medialer, qualitativer und inhaltlicher Hinsicht (Bräu und Schwerdt 2005; Brüning und Saum 2010) umsetzen. Dies kann sowohl für Schülergruppen, im Sinne eines ,flexible grouping“ (Rytivaara 2011), als auch für einzelne Schülerinnen und Schüler erfolgen. Da die konsequente Umsetzung einer solch umfassenden Differenzierung in einem klassisch lehrerzentrierten Unterrichtsarrangement an seine Grenzen stößt, wird eine Öffnung des Unterrichts notwendig (Brüning und Saum 2010; Kallweit 2015), das heißt eine Flexibilisierung 
von Raum, Zeit und Sozialform sowie eine Bereitstellung unterschiedlicher Lernund Unterrichtsmethoden. Somit kommen in einem individuell fördernden Unterricht neben Instruktionen durch die Lehrkraft verstärkt kooperative und individualisierte Lernformen zum Tragen (Trautmann und Wischer 2007). Auch wenn damit eine Dezentralisierung des Unterrichts einhergeht, ist es wichtig, dass dies nicht zu einer „Vereinzelung“ von Lernprozessen führt, sondern der „Klassenunterricht den Drehund Angelpunkt zur Planung, Verarbeitung, Zusammenführung und Auswertung unterschiedlicher Zugangs-, Lösungs- und Erkenntniswege“" (Scholz 2007, S. 21, s. a. Corno 2008) darstellt. Es geht also um ,,das Verbinden und Verbünden der Schüler/ innen in einer Weise, dass sie ihre fachliche wie soziale Anschlussfähigkeit in der Klassengemeinschaft wahren können“" (Klippert 2010, S. 219), und damit um die richtige Balance zwischen Individualisierung und Gemeinschaft (Lütje-Klose 2011).

Durch die Methoden der Binnendifferenzierung und durch die Öffnung des Unterrichts kommt es im Vergleich zu einem Unterricht, in dem alle Schülerinnen und Schüler das gleiche Unterrichtsangebot durch die Lehrkraft erhalten, zu einer veränderten Sichtstruktur. Die Tiefenstrukturen des Unterrichts im Sinne der drei Basisdimensionen von Unterrichtsqualität dürfen dabei jedoch nicht aus dem Blick geraten. So gilt auch für einen geöffneten und dezentralisierten Unterricht, dass die Steuerung und Strukturierung des Unterrichtsgeschehens weiterhin durch die Lehrkraft erfolgen muss und die Schülerinnen und Schüler nicht sich selbst überlassen werden können. Im Allgemeinen brauchen dabei Leistungsschwache mehr Struktur und Anleitung durch die Lehrkraft als Leistungsstärkere (Corno und Snow 1986). Eine konstruktive Unterstützung sollte durch das Eingehen auf die individuellen Bedürfnisse der Schülerinnen und Schüler relativ leicht umzusetzen sein.

Die größte Herausforderung stellt vermutlich die kognitive Aktivierung dar. In der Theorie müsste ein adaptiver Unterricht zu der bestmöglichen kognitiven Aktivierung der einzelnen Schülerinnen und Schüler führen, da sich jeder bzw. jede in seiner bzw. ihrer jeweiligen Zone der proximalen Entwicklung befinden sollte. Die Öffnung und Dezentralisierung des Unterrichts birgt jedoch die Gefahr, dass sich die Lehrkraft aufgrund der gestiegenen Komplexität des Unterrichtsgeschehens allein auf die Orchestrierung der verschiedenen Aktivitäten der Schülerinnen und Schüler konzentriert. Wichtig ist jedoch, dass die Lehrkraft eine tiefergehende Auseinandersetzung mit dem Unterrichtsgegenstand ermöglicht, indem sie zum Beispiel anregende Fragen stellt, auf Wichtiges hinweist, Zusammenhänge herstellt oder die Lernenden mit neuen Impulsen und Ideen konfrontiert. Eine gute und sorgfältige Unterrichtsplanung und Unterrichtsvorbereitung ist dafür zentral (Lipowsky und Lotz 2015).

Schließlich muss ein individuell fördernder Unterricht auch mit einer Ausdifferenzierung der Lernziele einhergehen (Vock und Gronostaj 2017). Hier bieten die Bildungsstandards mit ihrer Unterscheidung in Mindeststandards, Regelstandards und Optimalstandards eine hilfreiche Orientierung für Lehrkräfte, um die Lernziele für die einzelnen Schülerinnen und Schüler festzulegen und sicherzustellen, dass alle über bestimmte Basiskompetenzen verfügen. 


\section{Implikationen der vorgeschlagenen Konzeption}

Im Folgenden sollen Implikationen der im vorangegangenen Abschnitt vorgeschlagenen Konzeption zu individueller Förderung für deren Umsetzung in der Schulpraxis und für deren empirische Untersuchung erörtert und diskutiert werden.

\subsection{Implikationen für die Umsetzung von individueller Förderung in der Schulpraxis}

Auch wenn individuelle Förderung in aller Munde ist, stellt sich die Frage, wie verbreitet unterrichtsbezogene individuelle Förderung im Sinne der vorgeschlagenen Konzeption in der Schul- und Unterrichtspraxis ist. Hier zeichnet sich trotz der geringen empirischen Befundlage ein recht eindeutiges Bild ab: Während einzelne Unterrichtspraktiken, die mit einem individuellen fördernden Unterricht einhergehen, punktuell Einzug in die Unterrichtspraxis gefunden haben, scheint eine Gesamtunterrichtsstrategie zu individueller Förderung nur an äußerst wenigen Schulen zu existieren (Martschinke und Kammermeyer 2003; Bräu 2005; Ratzki 2005; Oelkers 2009; Maykus et al. 2011; Behrensen et al. 2012). Diese Schulen, bei denen es sich häufig um Modell- oder Reformschulen handelt, machen jedoch deutlich, dass die Umsetzung von individueller Förderung im regulären Unterricht gelingen kann (Czerwanski 2006; Skischus und Waltenberg 2006; Thurn 2006; Ebel et al. 2011). Dies zeigen auch die Preisträgerschulen des Deutschen Schulpreises, die größtenteils einen Unterricht praktizieren, der an die individuellen Lernvoraussetzungen der Schülerinnen und Schüler angepasst ist (Schratz et al. 2012). Bemerkenswert ist auch, dass in solchen Schulen die Heterogenität der Schülerschaft häufig sogar noch absichtlich verstärkt wird, indem Jahrgänge gemischt, Kinder mit sonderpädagogischem Förderbedarf inkludiert und äußere Formen der Differenzierung abgeschafft werden (Skischus und Waltenberg 2006; Thurn 2006).

Solche erfolgreichen Praxisbeispiele dürfen jedoch nicht darüber hinwegtäuschen, dass es sich bei der Umsetzung von unterrichtsbezogener individueller Förderung um ein äußerst anspruchsvolles Vorhaben handelt, das große Herausforderungen mit sich bringt - sowohl für die einzelne Lehrkraft als auch für die gesamte Schule. Für die Lehrkraft stellt es eine höchst anspruchsvolle Aufgabe dar, nicht nur ein Unterrichtsangebot für alle Schülerinnen und Schüler zu machen, sondern die verschiedenen, parallel ablaufenden Lernprozesse der einzelnen Schülerinnen und Schüler im Blick zu behalten und ihre Aufmerksamkeit gerecht zu verteilen (Trautmann und Wischer 2011; Breidenstein 2014; Lipowsky und Lotz 2015). Vor diesem Hintergrund überrascht es nicht, dass Lehrkräfte und Lehramtsstudierende das Unterrichten in einer heterogenen Klasse als eine der größten Herausforderungen ihrer Arbeit empfinden, auf die sie sich nicht hinreichend vorbereitet fühlen (Reh 2005; Gebauer et al. 2013). Um die Gefahr der Überforderung und damit eine Verringerung der Unterrichtsqualität zu vermeiden, bedarf diese Art zu unterrichten gründlicher Aus- und Weiterbildung sowie langfristiger Schul- und Unterrichsentwicklung (Oelkers 2009; Klippert 2010; Fischer 2014). 
Im Rahmen solcher Bemühungen sollten nicht nur Lehrerkompetenzen entwickelt, sondern auch Einstellungen von Lehrkräften zum Umgang mit Heterogenität adressiert werden, da diese als prädiktiv für zukünftiges Unterrichtsverhalten gelten können (Gebauer und McElvany 2017). Auf Ebene der Schule ist davon auszugehen, dass die konsequente Umsetzung von individueller Förderung im Unterricht eine Reihe von strukturellen und schulorganisatorischen Veränderungen notwendig macht (Bräu 2005; Trautmann und Wischer 2007; Schratz und Westfall-Greiter 2010; Wischer 2014). Zu nennen sind hier sowohl Rahmenbedingungen des Unterrichts, wie beispielsweise Änderungen des 45-Minuten-Takts, als auch Veränderungen in der Kooperation und Zusammenarbeit zwischen Lehrkräften, zum Beispiel in Form von Team-Teachings. Insofern ist eine Umsetzung von individueller Förderung im regulären Unterricht nicht von heute auf morgen möglich, sondern es sind grundlegende und nachhaltige Veränderungen auf allen Ebenen des Bildungssystems notwendig.

\subsection{Implikationen für die empirische Untersuchung von individueller Förderung im Unterricht}

Trotz empirischer Befunde zu einzelnen Bestandteilen von individueller Förderung im Unterricht gibt es bislang kaum empirische Erkenntnisse über die Gelingensbedingungen und die Wirksamkeit eines adaptiven und individuell fördernden Unterrichts als Ganzem (s. a. Martschinke 2016; Rabenstein und Wischer 2016). Ein Grund dafür mag sein, dass erst wenige Schulen diese Art von Unterricht praktizieren, was die empirische Untersuchung im Rahmen von repräsentativen Zufallsstichproben die für die empirische Unterrichtsforschung typisch sind - schwierig macht (Dumont 2016). Darüber hinaus ist die Analyse eines dezentralisierten Unterrichts, in dem verschiedene Unterrichtsaktivitäten zur gleichen Zeit stattfinden und sich die Schülerinnen und Schüler mit unterschiedlichen Unterrichtsgegenständen beschäftigen, in studienadministrativer, methodischer und inhaltlicher Hinsicht extrem komplex (Rademacher 2017) und erfordert die Anpassung der üblichen Erhebungsverfahren zur Messung von Unterrichtsqualität. In Bezug auf die Videographie von Unterricht müssten im Rahmen einer Unterrichtsstunde nicht nur diverse, teils informelle Lehrer-Schüler-Interaktionen, sondern auch Interaktionen zwischen den Schülerinnen und Schülern erfasst werden. Damit einhergehend stellt sich die Frage, ob es dann überhaupt noch ein Maß für die drei Basisdimensionen der Unterrichtsqualität geben kann, oder ob dies nicht in Abhängigkeit der Unterrichtsaktivitäten oder der einzelnen Schülerinnen und Schüler differenziert betrachtet werden muss. Auch für die Erfassung der Unterrichtsqualität durch Schülerangaben könnten Anpassungen notwendig sein. So implizieren die meisten Fragebögen zur Messung der Unterrichtsqualität ein lehrerzentriertes Unterrichtskonzept (siehe z. B. Itembeispiele von Klieme und Rakoczy 2008) und sind somit nicht ohne Weiteres auf einen dezentralisierten Unterricht übertragbar.

Neben der Anpassung bestehender Verfahren müssen darüber hinaus neue Instrumente entwickelt werden, um den Kern von adaptivem Unterricht empirisch erfassen zu können: die Adaptivität zwischen Unterrichtsangebot und Lernvoraussetzungen der Schülerinnen und Schüler (s. a. Hertel 2014). Dies stellt eine besondere Heraus- 
forderung dar, da der Grad der Adaptivität nicht beobachtet werden kann, sondern letztlich nur die Lernenden selbst darüber Auskunft geben können, wie passend das Unterrichtsangebot für sie war. Gleichzeitig ist jedoch fraglich, ob Schülerinnen und Schüler überhaupt in der Lage sind, solch ein Urteil abzugeben. Eine Möglichkeit, möglichst reliable und valide Aussagen zu erhalten, könnte darin bestehen, Schülerinnen und Schüler mehrfach und möglichst unmittelbar nach einzelnen Unterrichtsstunden zu befragen. Dadurch wäre es gleichzeitig möglich, die kumulative und sequenzielle Natur von Lernprozessen (Boekaerts 2017) sowie intraindividuelle Unterschiede im Lernprozess der Lernenden besser abbilden zu können.

\section{Ein kritischer (Aus-)Blick}

Bei allen Überlegungen zu individueller Förderung gilt es jedoch im Blick zu behalten, dass die hohe Bedeutung der individuellen Potenzialentfaltung und die Betonung von Individualität und Selbstbestimmung für die Entwicklung von Kindern letztlich auch ein Ausdruck der kulturellen Normen und Werte unserer eher individualistisch geprägten Gesellschaft darstellt (Hofstede 1986; Keller 2007). Es ist nicht ohne Weiteres davon auszugehen, dass diese Werte von allen Mitgliedern einer kulturell heterogenen Gemeinschaft mit der gleichen Selbstverständlichkeit geteilt und gelebt werden, wie es der vorherrschende Diskurs nahelegt. Wenn wir mit individueller Förderung eine Antwort auf die Frage nach dem Umgang mit einer heterogenen Schülerschaft geben und Ungleichheiten reduzieren wollen, muss Sorge dafür getragen werden, dass hierdurch nicht auf einer anderen Ebene neue Ungleichheiten produziert werden - wenn etwa unterschiedliche Werte und Normen nicht berücksichtigt werden. Dies ist insbesondere vor dem Hintergrund zunehmender kultureller Heterogenität im Schulsystem von großer Bedeutung.

In Bezug auf die Frage, ob durch individuelle Förderung mehr Chancengerechtigkeit hergestellt werden kann, gilt es eine Reihe von normativen Fragen zu adressieren (Martschinke 2016). So könnte es beispielsweise sein, dass sich die Schere zwischen leistungsstarken und leistungsschwachen Schülerinnen und Schülern weiter öffnet, wenn jeder und jede entsprechend seinem und ihrem individuellen Potenzial gefördert wird (Lipowsky und Lotz 2015). Schließlich darf auch nicht aus den Augen verloren werden, dass unser Schulsystem nicht nur eine Förder-, sondern auch eine Selektionsfunktion hat, was zu bestimmten Elementen eines individuell fördernden Unterrichts im Widerspruch stehen kann.

Auch wenn es sich bei dem Begriff ,individuelle Förderung“ in gewisser Weise um einen „neuen Schlauch“ für seit Langem bestehende Ideen und Konzepte handelt, ist der aktuelle Diskurs in Politik und Praxis eine Chance, sich vertieft damit auseinanderzusetzen, wie individuelle Förderung im Rahmen des regulären Unterrichts aussehen und umgesetzt werden kann. Vor diesem Hintergrund ist mit dem vorliegenden Beitrag die Hoffnung verbunden, die teils parallel stattfindenden Diskurse in Politik, Praxis und Wissenschaft näher zusammenzubringen und das Fundament für eine verstärkte wissenschaftliche Auseinandersetzung mit und empirische Untersuchung von individueller Förderung im Unterricht zu legen. 
Open Access Dieser Artikel wird unter der Creative Commons Namensnennung 4.0 International Lizenz (http://creativecommons.org/licenses/by/4.0/deed.de) veröffentlicht, welche die Nutzung, Vervielfältigung, Bearbeitung, Verbreitung und Wiedergabe in jeglichem Medium und Format erlaubt, sofern Sie den/die ursprünglichen Autor(en) und die Quelle ordnungsgemäß nennen, einen Link zur Creative Commons Lizenz beifügen und angeben, ob Änderungen vorgenommen wurden.

\section{Literatur}

Ahnen, D. (2004). Individuelle Förderung - Grundlage für ein Bildungssystem mit Zukunft, Fördern und Fordern - Herausforderung an Schule und Lehrkräfte. Bonn. http://www.kmk.org/fileadmin/ Dateien/pdf/PresseUndAktuelles/2004/Rede_Praesidentin_Foerdern_und_Fordern.pdf. Zugegriffen: 22. Sept. 2016.

Alfieri, L., Brooks, P. J., \& Aldrich, N. J. (2011). Does discovery-based instruction enhance learning? Journal of Educational Psychology, 103, 1-18.

Allen, M.H., Matthews, C.E., \& Parsons, S. A. (2013). A second-grade teacher's adaptive teaching during an integrated science literacy unit. Teaching and Teacher Education, 35, 114-125.

Andrade, H. L., \& Cizek, G. J. (Hrsg.) (2009). Handbook of formative assessment. New York: Routledge, Taylor and Francis.

Arnold, K.-H., \& Richert, P. (2008). Unterricht und Förderung: Die Perspektive der Didaktik. In K.-H. Arnold, O. Graumann, \& A. Rakhkochkine (Hrsg.), Handbuch Förderung (S. 26-35). Weinheim: Beltz.

Bangert, R.L., Kulik, J.A., \& Kulik, C.-L.C. (1983). Individualized systems of instruction in secondary schools. Review of Educational Research, 53, 143-158.

Beck, E., Baer, M., Guldimann, T., Bischoff, S., Brühwiler, C., Müller, P., Niedermann, R., Rogalla, M., \& Vogt, F. (2008). Adaptive Lehrkompetenz. Analyse und Struktur, Veränderbarkeit und Wirkung handlungssteuernden Lehrerwissens. Münster: Waxmann.

Behrensen, B., \& Solzbacher, C. (2012). Individuelle Förderung in KiTa und Grundschule (Nifbe-Themenheft: Nr. 5). Osnabrück: Nifbe-Eigenverlag.

Behrensen, B., Sauerhering, M., \& Solzbacher, C. (2012). Ausgewählte Ergebnisse einer empirischen Studie zu Sichtweisen und Erfahrungen von Grundschullehrkräften zu individueller Förderung. In C. Solzbacher, S. Müller-Using, \& I. Doll (Hrsg.), Ressourcen stärken! Individuelle Förderung als Herausforderung für die Grundschule (S. 3-16). Köln: Link.

Behrensen, B., Gläser, E., \& Solzbacher, C. (Hrsg.) (2015). Fachdidaktik und individuelle Förderung in der Grundschule. Perspektiven auf Unterricht in heterogenen Lerngruppen. Baltmannsweiler: SchneiderVerlag Hohengehren.

Bildungsrat, D. (1970). Empfehlungen der Bildungskommission. Strukturplan für das Bildungswesen. Stuttgart: Klett.

Black, P., \& Wiliam, D. (1998). Assessment and classroom learning. Assessment in Education, 5.

Black, P., \& Wiliam, D. (2009). Developing the theory of formative assessment. Educational Assessment, Evaluation and Accountability, 21, 5-32.

Blaes, C., Anus, S., Kallweit, I., Naeve, S., \& Melle, I. (2012). Individuelle Förderung im Chemieunterricht. Der mathematisch und naturwissenschaftliche Unterricht (MNU), 65, 293-300.

Boekaerts, M. (1999). Self-regulated learning: where we are today. International Journal of Educational Research, 31, 445-457.

Boekaerts, M. (2017). Cognitive load and self-regulation: attempts to build a bridge. Learning and Instruction, 51, 90-97.

Boekaerts, M., \& Corno, L. (2005). Self-regulation in the classroom: a perspective on assessment and intervention. Applied Psychology: An international Review, 54, 199-231.

Boekaerts, M., Pintrich, P.R., \& Zeidner, M. (Hrsg.) (2000). Handbook of self-regulation. San Diego: Academic Press.

Bohl, T., \& Kucharz, D. (2010). Offener Unterricht heute. Weinheim: Beltz.

Bohl, T., Batzel, A., \& Richey, P. (2011). Öffnung - Differenzierung - Individualisierung - Adaptivität: Charakteristika, didaktische Implikationen und Forschungsbefunde verwandter Unterrichtskonzepte zum Umgang mit Heterogenität. Schulpädagogik Heute, 2, 40-69.

Bönsch, M. (1986). Adaptiver Unterricht. Zeitschrift für internationale erziehungs- und sozialwissenschaftliche Forschung, 3, 73-85.

Bönsch, M. (2004). Differenzierung in Schule und Unterricht. München: Oldenbourg Schulbuchverlag. 
Bransford, J.D., Brown, A. L., \& Cocking, R. R. (2000). How people learn: brain, mind, experience, and school (2. Aufl.). Washington, DC: National Academy Press.

Bräu, K. (2005). Individualisierung des Lernens - Zum Lehrerhandeln bei der Bewältigung eines Balanceproblems. In K. Bräu \& U. Schwerdt (Hrsg.), Heterogenität als Chance. Vom produktiven Umgang mit Gleichheit und Differenz in der Schule (S. 129-150). Münster: LIT.

Bräu, K., \& Schwerdt, U. (Hrsg.) (2005). Heterogenität als Chance. Vom produktiven Umgang mit Gleichheit und Differenz in der Schule. Münster: Lit.

Breidenstein, G. (2014). Die Individualisierung des Lernens unter den Bedingungen der Institution Schule. In B. Kopp, S. Martschinke, M. Munser-Kiefer, M. Haider, E.-M. Kirschhock, G. Ranger, \& G. Renner (Hrsg.), Individuelle Förderung und Lernen in der Gemeinschaft (Jahrbuch Grundschulforschung: Bd. 17, S. 35-50). Wiesbaden: Springer VS.

Breidenstein, G., \& Rademacher, S. (2016). Individualisierung und Standardisierung von Unterrichtszeit. Empirische Beobachtungen und Analysen. In K. Rabenstein \& B. Wischer (Hrsg.), Individualisierung schulischen Lernens (S. 16-32). Seelze: Klett.

Breidenstein, G., \& Rademacher, S. (2017). Individualisierung und Kontrolle. Empirische Studien zum geöffneten Unterricht in der Grundschule. Wiesbaden: Springer VS.

Brüning, L., \& Saum, T. (2010). Individualisierung und Differenzierung - aber wie? Kooperatives Lernen erschließt neue Zugänge. Pädagogik, 62, 12-15.

Burton, D. (2007). Psycho-pedagogy and personalised learning. Journal of Education for Teaching, 33, 5-17.

Butler, D.L., \& Winne, P.H. (1995). Feedback and self-regulated learning: a theoretical synthesis. Review of Educational Research, 65, 245-281.

Campbell, R.J., Robinson, W., Neelands, J., Hewston, R., \& Mazzoli, L. (2007). Personalised learning: ambiguities in theory and practice. British Journal of Educational Studies, 55, 135-154.

Clarke, J.H. (2013). Personalized learning: student-designed pathways to high school graduation. Thousand Oaks: Corwin.

Connor, C. M., Morrison, F. J., \& Petrella, J. N. (2004). Effective reading comprehension instruction: Examining child by instruction interactions. Journal of Educational Psychology, 96, 682-698.

Connor, C.M., Piasta, S.B., Fishman, B., Glasney, S., Schatschneider, C., Crowe, E., \& Morrison, F. (2009). Individualizing student instruction precisely: effects of child $\mathrm{x}$ instruction interactions on first graders' literacy development. Child Development, 80, 77-100.

Constas, M. A., \& Sternberg, R. J. (2006). Translating theory and research into educational practice: developments in content domains, large scale reform, and intellectual capacity. Mahwah: Lawrence Erlbaum.

Corno, L. (2008). On teaching adaptively. Educational Psychologist, 43, 161-173.

Corno, L., \& Snow, R.E. (1986). Adapting teaching to individual differences among learners. In M.C. Wittrock (Hrsg.), Handbook of research on teaching (S. 605-629). London: Macmillan.

De Corte, E. (2010). Historical developments in the understanding of learning. In H. Dumont, D. Istance, \& F. Benavides (Hrsg.), The nature of learning. Using research to inspire practice (S. 35-68). Paris: OECD.

Courtis, S. A. (1938). Contributions of research to the individualization of instruction. National Society for the Study of Education, 37, 201-210.

Cronbach, L. J. (1967). How can instruction be adapted to individual differences? In R. M. Gagné (Hrsg.), Learning and individual differences (S. 12-58). Columbus: Merrill.

Cronbach, L. J., \& Snow, R.E. (1977). Aptitudes and instructional methods: a handbook for research on interactions. New York: Irvington.

Czerwanski, A. (2006). Voraussetzungen für Individualisierung schaffen. Von der Haltung der Lehrenden bis zu den Kompetenzen der Lernenden. Pädagogik, 1, 10-14.

Decristan, J., Hondrich, A.L., Büttner, G., Hertel, S., Klieme, E., Kunter, M., Lühken, A., Adl-Armini, K., Djakovic, S.-K., Mannel, S., Naumann, A., \& Hardy, I. (2015a). Impact of additional guidance in science education on primary students' conceptual understanding. The Journal of Educational Research, 108, 358-370.

Decristan, J., Klieme, E., Kunter, M., Hochweber, J., Büttner, G., Fauth, B., Hondrich, A.L., Rieser, S., Hertel, S., \& Hardy, I. (2015b). Embedded formative assessment and classroom process quality: How do they interact in promoting students' science understanding? American Educational Research Journal, 52(6), 1133-1159.

Decristan, J., Hardy, I., Klieme, E., Büttner, Hertel, S., Kunter, M., \& Lühken, A. (2017). Individuelle Förderung und adaptive Lerngelegenheiten im Grundschulunterricht. Einführung. In U. Hartmann, M. 
Hasselhorn, \& A. Gold (Hrsg.), Entwicklungsverläufe vestehen - Kinder mit Bildungsrisiken wirksam fördern (S. 312-326). Stuttgart: Kohlhammer.

Diack, A. (2004). Innovation and personalised learning. Education Review, 18, 49-55.

Diesbergen, C. (2010). Der Konstruktivismus - eine geeignete Grundlagentheorie zum Umgang mit Heterogenität im Unterricht? In H. U. Grunder \& A. Gut (Hrsg.), Zum Umgang mit Heterogenität in Schule und Gesellschaft. Bd. II: Chancen und Problemlagen (S. 77-95). Baltmannsweiler: Schneider.

Dräger, J. (2009). Individuelle Förderung für ein faires und leistungsstarkes Schulsystem. In B. Stiftung (Hrsg.), Heterogenität und Bildung. Individuelle Förderung in Deutschland - Hindernisse und Herausforderungen (S. 4-8). Gütersloh: Bertelsmann Stiftung.

Dumont, H. (2016). Die empirische Untersuchung von individueller Förderung als Perspektive für die Unterrichtsqualitätsforschung. In N. McElvany, W. Bos, H. G. Holtappels, M. Gebauer, \& F. Schwabe (Hrsg.), Bedingungen und Effekte guten Unterrichts - Aktueller Stand und Perspektiven der Unterrichtsforschung (S. 107-116). Münster: Waxmann.

Dumont, H., Istance, D., \& Benavides, F. (Hrsg.) (2010). The nature of learning. Using research to inspire practice. Paris: OECD.

Ebel, C., Hollenbach, N., \& Müncher, A. (2011). Heterogenität als Herausorderung für Schule und Unterricht. Was ,individuelle Förderung“ in der Unterrichtspraxis bedeutet und wie sich Schulen - trotz schwieriger Rahmenbedingungen - auf den Weg machen können. Podium Schule, 1.11, 1-3.

Eckert, E. (2004). Individuelles Fördern. In H. Mayer (Hrsg.), Was ist guter Unterricht? (S. 86-103). Berlin: Cornelsen.

Fischer, C. (2014). Individuelle Förderung als schulische Herausforderung. Berlin: Friedrich-Ebert-Stiftung.

Forum Bildung (2001). Empfehlungen des Forum Bildung. http://www.blk-bonn.de/papers/forumbildung/ergebnisse-fb-band01.pdf. Zugegriffen: 22. Sept. 2016.

Gebauer, M.M., \& McElvany, N. (2017). Zur Bedeutsamkeit unterrichtsbezogener heterogenitätsspezifischer Einstellungen angehender Lehrkräfte für zukünftiges Unterrichtsverhalten. Psychologie in Erziehung und Unterricht, 64(3), 163-180.

Gebauer, M. M., McElvany, N., \& Emmer, E. T. (2013). Einstellungen von Lehramtsanwärterinnen und Lehramtsanwärtern zum Umgang mit heterogenen Schülergruppen in Schule und Untericht. In N. MElvany (Hrsg.), Jahrbuch der Schulentwicklung. Bd. 17: Sprachliche, kulturelle und soziale Heterogenität in der Schule als Herausforderung und Chance der Schulentwicklung (S. 191-216). Weinheim: Beltz Juventa.

Glaser, R. (1972). Individuals and learning: the new aptitudes. Educational Researcher, 1, 5-13.

Glaser, R. (1977). Adaptive education: individual diversity and learning. New York: Holt McDougal.

Graumann, O. (2002). Gemeinsamer Unterricht in heterogenen Gruppen. Bad Heilbrunn: Klinkhardt.

Graumann, O. (2008). Förderung und Heterogenität: Die Perspektive der Schulpägagogik. In K.-H. Arnold, O. Graumann, \& A. Rakhkochkine (Hrsg.), Handbuch Förderung. Grundlagen, Bereiche und Methoden der individuellen Förderung von Schülern. Weinheim: Beltz.

Gutierrez, R., \& Slavin, R. (1992). Achievement effects of the nongraded elementary school: a best evidence synthesis. Review of Educational Research, 62, 333-376.

Haag, L., \& Streber, D. (2014). Individuelle Förderung. Eine Einführung in Theorie und Praxis. Weinheim: Beltz.

Hardy, I. (2017). Individuelle Förderung von Lernprozessen. Einführung. In U. Hartmann, M. Hasselhorn, \& A. Gold (Hrsg.), Entwicklungsverläufe vestehen - Kinder mit Bildungsrisiken wirksam fördern (S. 267-279). Stuttgart: Kohlhammer.

Hardy, I., Jonen, A., Möller, K., \& Stern, E. (2006). Effects of instructional support within constructivist learning environments for elementary school students' understanding of "floating and sinking“. Journal of Educational Psychology, 98, 307-326.

Hardy, I., Hertel, S., Kunter, M., Klieme, E., Warwas, J., Büttner, G., \& Lühken, A. (2011). Adaptive Lerngelegenheiten in der Grundschule: Merkmale, methodisch-didaktische Schwerpunktsetzungen und erforderliche Lehrerkompetenzen. Zeitschrift für Pädagogik, 57, 819-833.

Hartmann, U., Hasselhorn, M., \& Gold, A. (Hrsg.) (2017). Entwicklungsverläufe verstehen - Kinder mit Bildungsrisiken wirksam fördern. Stuttgart: Kohlhammer.

Hasselhorn, M., Decristan, J., \& Klieme, E. (2018). Individuelle Förderung. In Köller, O., Hasselhorn, M., Hesse, F., Maaz, K., Schrader, J., Solga, H., Spieß, C. K., \& Zimmer, K. (Hrsg.), Das Bildungswesen in Deutschland. Bestand und Potenziale. Bad Heilbrunn: Klinkhardt.

Hattie, J. (2009). Visible learning. A synthesis of over 800 meta-analyses relating to achievement. London: Routledge. 
Helmke, A. (2009). Unterrichtsqualität und Lehrerprofessionalität. Diagnose, Evaluation und Verbesserung des Unterrichts. Seelz: Kallmeyer.

Helmke, A. (2013). Individualisierung: Hintergrund, Missverständnisse, Perspektiven. Pädagogik, 65, 34-37.

Helmke, A., \& Weinert, F.E. (1997). Bedigungsfaktoren schulischer Leistung. In F. E. Weinert (Hrsg.), Psychologie des Unterrichts und der Schule: Enzyklopädie der Psychologie (S. 71-176). Göttingen: Hogrefe.

Hertel, S. (2014). Adaptive Lerngelegenheiten in der Grundschule: Merkmale, methodisch-didaktische Schwerpunktsetzungen und erforderliche Lehrerkompetenzen. In B. Kopp, S. Martschinke, M. Munser-Kiefer, M. Haider, E.-M. Kirschhock, G. Ranger, \& G. Renner (Hrsg.), Individuelle Förderung und Lernen in der Gemeinschaft (Jahrbuch Grundschulforschung: Bd. 17, S. 19-34). Wiesbaden: Springer VS.

Hertel, S., Fingerle, M., \& Rohlfs, C. (2016). Gestaltung adaptiver Lerngelegenheiten in der Schule. In K. Rabenstein \& B. Wischer (Hrsg.), Individualisierung schulischen Lernens. Mythos oder Königsweg? (S. 64-75). Seelze-Velber: Klett Kallmeyer.

Hess, M., \& Lipowsky, F. (2017). Lernen individualisieren und Unterrichtsqualität verbessern. In F. Heinzel \& K. Koch (Hrsg.), Individualisierung im Grundschulunterricht. Anspruch, Realisierung und Risiken (S. 23-31). Wiesbaden: Springer.

Heymann, H.W. (2010). Binnendifferenzierung - eine Utopie? Pädagogischer Anspruch, didaktisches Handwerk, Realisierungschancen. Pädagogik, 62, 6-10.

Hofstede, G. (1986). Cultural differences in teaching and learning. International Journal of Intercultural Relations, 10, 301-320.

Höhmann, K. (2009). Individualisierung: nicht nur eine Frage von Methode und Haltung. In R. Hinz \& R. Walthes (Hrsg.), Heterogenität in der Grundschule. Den pädagogischen Alltag erfolgreich bewältigen (S. 212-217). Weinheim: Beltz.

Horak, V.M. (1981). A meta-analysis of research findings on individualized instruction in mathematics. Journal of Educational Research, 74, 249-253.

Johnson, M. (2004). Personalised learning. New Economy, 11, 224-228.

Kallweit, I. (2015). Effektivität des Einsatzes von Selbsteinschätzungsbögen im Chemieunterricht der Sekundarstufe I. Individuelle Förderung durch selbstreguliertes Lernen. Berlin: Logos.

Kalyuga, S. (2007). Expertise reversal effect and its implications for learner-tailored instruction. Educational Psychological Review, 19, 509-539.

Keller, H. (2007). Socialization for competence: cultural models of infancy. Human Development, 46, $288-311$.

Kingston, N., \& Nash, B. (2011). Formative assessment: a meta-analysis and a call for research. Educational Measurement: Issues and Practice, 30, 28-37.

Kirschner, P. A., Sweller, J., \& Clark, R.E. (2006). Why minimal guidance during instruction does not work: an analysis of the failure of constructivist, discovery, problem-based, experiential, and inquirybased teaching. Educational Psychologist, 41, 75-86.

Klafki, W., \& Stöcker, H. (1976). Innere Differenzierung des Unterrichts. Zeitschrift für Pädagogik, 22, 497-523.

Klieme, E. (2006). Empirische Unterrichtsforschung: aktuelle Entwicklungen, theoretische Grundlagen und fachspezifische Befunde. Zeitschrift für Pädagogik, 52, 765-773.

Klieme, E., \& Rakoczy, K. (2008). Empirische Unterrichtsforschung und Fachdidaktik. Outcome-orientierte Messung und Prozessqualität des Unterrichts. Zeitschrift für Pädagogik, 54, 222-237.

Klieme, E., \& Warwas, J. (2011). Konzepte der individuellen Förderung. Zeitschrift für Pädagogik, 57, $805-818$.

Klippert, H. (2010). Heterogenität im Klassenzimmer. Wie Lehrkräfte effektiv und zeitsparend damit umgehen können. Weinheim: Beltz.

KMK (2010) = Kultusministerkonferenz. (Hrsg.) (2010). Förderstrategie für leistungsschwache Schülerinnen und Schüler. Beschluss der Kultusministerkonferenz vom 04.03.2010. http://www.kmk.org/ fileadmin/Dateien/veroeffentlichungen_beschluesse/2010/2010_03_04-FoerderstrategieLeistungsschwaechere.pdf. Zugegriffen: 22. Sept. 2017.

KMK = Kultusministerkonferenz. (Hrsg.) (2015). Förderstrategie für leistungstarke Schülerinnen und Schüler. Beschluss der Kultusministerkonferenz vom 11.06.2015. http://www.kmk.org/fileadmin/ Dateien/pdf/350-KMK-TOP-011-Fu-Leistungsstarke_-_neu.pdf. Zugegriffen: 22. Sept. 2017.

Krammer, K. (2009). Individuelle Lernunterstützung in Schülerarbeitsphasen. Eine videobasierte Analyse des Unterstützungsverhaltens von Lehrpersonen im Mathematikunterricht. Münster: Waxmann. 
Kulik, C.-L.C., \& Kulik, J. A. (1982). Effects of ability grouping on secondary school students: a metaanalysis of evaluation findings. American Educational Research Journal, 19, 415-428.

Kunter, M., \& Trautwein, U. (2013). Psychologie des Unterrichts. Paderborn: Ferdinand Schöningh.

Kunze, I. (2008). Begründungen und Problembereiche individueller Förderung in der Schule - Vorüberlegungen zu einer empirischen Untersuchung. In I. Kunze \& C. Solzbacher (Hrsg.), Individuelle Förderung in der Sekundarstufe I und II (S. 13-25). Baltmannsweiler: Schneider-Verlag Hohengehren.

Lewis, R. (1986). What is open learning? Open Learning: The Journal of Open, Distance and E-Learning, $1,5-10$.

Lipowsky, F., \& Lotz, M. (2015). Ist Individualisierung der Königsweg zum Lernen? Eine Auseinandersetzung mit Theorien, Konzepten und empirischen Befunden. In F. Mehlhorn, F. Schulz, \& K. Schöppe (Hrsg.), Begabungen entwickeln and Kreativität fördern (S. 155-219). München: kopaed.

Looney, J. (2005). Formative assessment: improving learning in secondary classrooms. Paris: OECD.

Lotz, M. (2015). Kognitive Aktivierung im Leseunterricht der Grundschule: Eine Videostudie zur Gestaltung und Qualität von Leseübungen im ersten Schuljahr. Wiesbaden: Springer.

Lou, Y., Abrami, P.C., Spence, J.C., Poulsen, C., Chambers, B., \& d'Apollonia, S. (1996). Within-class grouping: a meta-analysis. Review of Educational Research, 66, 449-521.

Lütje-Klose, B. (2011). Müssen Lehrkräfte ihr didaktisches Handwerk verändern? Inklusive Didaktik als Herausforderung für den Unterricht. Lernende Schule, 55, 13-15.

Maaz, K., Baumert, J., \& Trautwein, U. (2009). Genese sozialer Ungleichheit im institutionellen Kontext der Schule: wo entsteht und vergrößert sich soziale Ungleichheit. In J. Baumert, K. Maaz, \& U. Trautwein (Hrsg.), Bildungsentschiedungen (Zeitschrift für Erziehungswissenschaft: Sonderheft 12, S. 11-46). Wiesbaden: VS.

Martschinke, S. (2016). Facetten adaptiven Unterrichts aus der Sicht der Unterrichtsforschung. In K. Liebers, B. Landwehr, A. Marquardt, \& K. Schlotter (Hrsg.), Lernprozessbegleitung und adaptives Lernen in der Grundschule. Forschungsbezogene Beiträge (S. 15-32). Wiesbaden: Springer.

Martschinke, S., \& Kammermeyer, G. (2003). Jedes Kind ist anders. Jede Klasse ist anders. Zeitschrift für Erziehungswissenschaft, 6(2), 257-275.

Mayer, R.E. (2004). Should there be a three-strikes rule against pure discovery learning? American Psychologist, 59, 14-19.

Maykus, S., Böttcher, W., Liesegang, T., \& Altermann, A. (2011). Individuelle Förderung in der Ganztagsschule. Theoretisch-empirische Reflexionen zu Anspruch und Konsequenzen eines (sozial-)pädagogischen Programms im professionellen Handeln. In L. Stecher, H.-H. Krüger, \& T. Rauschenbach (Hrsg.), Ganztagsschule - Neue Schule? (S. 125-142). Wiesbaden: VS.

McTighe, J., \& Brown, J. L. (2005). Differentiated instruction and educational standards. Theory Into Practice, 44, 234-244.

Van Merriënboer, J., \& Sweller, J. (2005). Cognitive load theory and complex learning: recent developments and future directions. Educational Psychology Review, 17, 147-177.

Mulkey, L.M., Catsambis, S., Steelman, L.C., \& Crain, R.L. (2005). The long-term effects of ability grouping in mathematics: a national investigation. Social Psychology of Education, 8, 137-177.

Oelkers, J. (2009). Barrieren für individuelle Förderung im Bildungssystem und ihre Bearbeitung. Eine Expertise für die Bertelsmann Stiftung. In B. Stiftung (Hrsg.), Heterogenität und Bildung. Individuelle Förderung in Deutschland - Hindernisse und Herausforderungen (S. 9-35). Gütersloh: Bertelsmann Stiftung.

Otto, B., Perels, F., \& Schmitz, B. (2015). Selbstreguliertes Lernen. In H. Reinders, H. Ditton, C. Gräsel, \& B. Gniewosz (Hrsg.), Empirische Bildungsforschung. Gegenstandsbereiche (S. 41-53). Wiesbaden: Springer.

Paradies, L., \& Linser, H. J. (2008). Differenzieren im Unterricht. Berlin: Cornelson Scriptor.

Paradies, L., Wester, F., \& Greving, J. (2010). Individualisieren im Unterricht. Erfolgreich Kompetenzen vermitteln. Berlin: Cornelson Scriptor.

Paris, S. G., \& Paris, A.H. (2001). Classroom applications of research on self-regulated learning. Educational Psychologist, 36, 89-101.

Parsons, S. A. (2012). Adaptive teaching in literacy instruction: case studies of two teachers. Journal of Literacy Research, 44, 149-170.

Peschel, F. (2002). Offener Unterricht - Idee, Realität, Perspektive und ein praxiserprobtes Konzept zur Diskussion. Teil I: Allgemeindidaktische Überlegungen. Baltmannsweiler: Schneider-Verlag Hohengehren.

Pianta, R.C., \& Hamre, B.K. (2009). Conceptualization, measurement and improvement of classroom processes: standardized observation can leverage capacity. Educational Researcher, 38, 109-119. 
Pintrich, P. R. (2000). The role of goal orientation in self-regulated learning. In M. Boekaerts, P. R. Pintrich, \& M. Zeidner (Hrsg.), Handbook of self-regulation (S. 451-502). San Diego: Academic Press.

Van de Pol, J., Volman, M., \& Beishuizen, J. (2010). Scaffolding in teacher-student interaction: a decade of research. Educational Psychology Review, 22, 271-296.

Van de Pol, J., Volman, M., Oort, F., \& Beishuizen, J. (2015). The effects of scaffolding in the classroom: support contingency and student independent working time in relation to student achievement, task effort and appreciation of support. Instructional Science, 43, 615-641.

Rabenstein, K., \& Wischer, B. (2016). Forschung zur Individualisierung im und von Unterricht. In K. Rabenstein \& B. Wischer (Hrsg.), Individualisierung schulischen Lernens (S. 6-15). Seelze: Klett.

Rademacher, S. (2017). Zur Praxis des individualisierten Grundschulunterrichts. In F. Heinzel \& K. Koch (Hrsg.), Individualisierung im Grundschulunterricht. Anspruch, Realisierung und Risiken (S. 23-31). Wiesbaden: Springer.

Randi, J., \& Corno, L. (2005). Teaching and learner variation. Pedagogy-Learning for Teaching. British Journal of Educational Psychology, Monograph Series II, 3, 47-69.

Ratzki, A. (2005). Pädagogik der Vielfalt im Licht internationaler Schulerfahrungen. In K. Bräu \& U. Schwerdt (Hrsg.), Heterogenität als Chance. Vom produktiven Umgang mit Gleichheit und Differenz. in der Schule (S. 37-52). Münster: LIT.

Reh, S. (2005). Warum fällt es Lehrerinnen und Lehrern so schwer, mit Heterogenität umzugehen? Historische und empirische Deutungen. Die deutsche Schule, 97(1), 76-86.

Reusser, K. (2015). Heterogene Lerngruppen unterrichten - maßgeschneiderte Angebote für kompetenzorientiertes Lernen. In C. Villiger \& U. Trautwein (Hrsg.), Zwischen Theorie und Praxis. Ansprüche und Möglichkeiten in der Lehrer(innen)bildung (S. 223-242). Münster: Waxmann.

Ricken, G. (2008). Förderung aus sonderpädagogischer Sicht. In K.-H. Arnold, O. Graumann, \& A. Rakhkochkine (Hrsg.), Handbuch Förderung. Grundlagen, Bereich eund Methoden der individuellen Förderung von Schülern (S. 74-83). Weinheim: Beltz.

Rytivaara, A. (2011). Flexible grouping as a means for classroom management in a heterogeneous classroom. European Educational Research Journal, 10, 118-128.

Sadler, D. R. (1989). Formative assessment and the design of instructional systems. Instructional Science, 18, 119-144.

Sawyer, R. K. (2006). The Cambridge handbook of the learning sciences. Cambridge: Cambridge University Press.

Schmitz, B. (2001). Self-Monitoring zur Unterstützung des Transfers einer Schulung in Selbstregulation für Studierende. Zeitschrift für Pädagogische Psychologie, 15, 181-197.

Schneider, M., \& Stern, E. (2010). The cognitive perspective on learning: ten cornerstone findings. In H. Dumont, D. Istance, \& F. Benavides (Hrsg.), The nature of learning. Using research to inspire practice (S. 82-100). Paris: OECD.

Schneuwly, G. (2014). Differenzierungskonzepte sichtbar gemacht. Eine qualitative Fallstudie zur inneren Differenzierung im Mathematikunterricht der Primarschulstufe. Münster: Waxmann.

Schnotz, W. (2016). Learning and instruction: a review of main research lines during recent decades. Zeitschrift für Erziehungswissenschaft, 19(1), 101-119.

Schofield, J. W. (2010). International evidence on ability grouping with curriculum differentiation and the achievement gap in secondary schools. Teachers College Record, 112, 1492-1528.

Scholz, I. (2007). Es ist normal, verschieden zu sein - Unterrichten in heterogenen Klassen. In I. Scholz (Hrsg.), Der Spagat zwischen Fördern und Fordern (S. 7-23). Göttingen: Vandenhoeck \& Ruprecht.

Schratz, M., \& Westfall-Greiter, T. (2010). Das Dilemma der Individualisierungsdidaktik. Plädoyer für personalisiertes Lernen in der Schule. Journal für Schulentwicklung, 1, 18-31.

Schratz, M., Pant, H.A., \& Wischer, B. (2012). Was für Schulen! Vom Umgang mit Vielfalt - Beispiele guter Praxis. Seelze: Klett.

Schwarzer, R., \& Steinhagen, K. (Hrsg.). (1975). Adaptiver Unterricht. Zur Wechselwirkung von Schülermerkmalen und Unterrichtsmethoden. München: Kösel.

Skischus, G., \& Waltenberg, B. (2006). Umgang mit Heterogenität lernen. Erfahrungen mit individuellen Zugängen zu Inhalt, Leistung und sozialem Verhalten. Pädagogik, 58, 44-47.

Slavin, R.E. (1987). Ability grouping and student achievement in elementary schools: a best-evidence synthesis. Review of Educational Research, 57, 293-336.

Slavin, R.E. (1990). Achievement effects of ability grouping in secondary schools: a best-evidence synthesis. Review of Educational Research, 60, 471-499.

Sliwka, A. (2012). Diveresität als Chance und als Ressource in der Gestaltung wirksamer Lernprozesse. In K. Fereidooni (Hrsg.), Das interkulturelle Lehrerzimmer. Perspektiven neuer deutscher Lehrkräfte auf den Bildungs- und Integrationsdiskurs (S. 169-176). Wiesbaden: VS. 
Snow, R. E. (1989). Aptitude-treatment interaction as a framework for research on individual differences. In P. Ackermann, R. J. Sternberg, \& R. Glaser (Hrsg.), Learning and Individual Differences (S. 13-59). New York: W. H. Freeman.

Sweller, J. (2003). Evolution of human cognitive architecture. In B. Ross (Hrsg.), The psychology of learning and motivation (Bd. 43, S. 215-266). San Diego: Academic Press.

Sweller, J., van Merrienboer, J., \& Paas, F. (1998). Cognitive architecture and instructional design. Educational Psychology Review, 10, 251-296.

Thurn, S. (2006). Individualisierung kann gelingen. Begriffliche Klärung, Erfahrungen, Gelingensbedingungen. Pädagogik, 1, 6-9.

Trautmann, M., \& Wischer, B. (2007). Individuell fördern im Unterricht. Was wissen wir über Innere Differenzierung? Pädagogik, 12, 44-48.

Trautmann, M., \& Wischer, B. (2008). Das Konzept der Inneren Differenzierung - eine vergleichende Analyse der Diskussion der 1970er Jahre mit dem aktuellen Heterogenitätsdiskurs. In M. A. Meyer, M. Prenzel, \& S. Hellekamps (Hrsg.), Perspektiven der Didaktik (Zeitschrift für Erziehungswissenschaft: Sonderheft 9, S. 159-172). Wiesbaden: VS.

Trautmann, M., \& Wischer, B. (2011). Heterogenität in der Schule. Eine kritische Einführung. Wiesbaden: VS.

Vock, M., \& Gronostaj, A. (2017). Umgang mit Heterogenität in Schule und Unterricht. Berlin: FriedrichEbert-Stiftung.

Vygotsky, L. (1963). Thought and language. Cambridge: MIT Press.

Wang, M.C. (1992). Adaptive education strategies: building on diversity. Baltimore: Brookes.

Wang, M.C., \& Lindvall, C. M. (1984). Individual differences and school learning environments. Review of Research in Education, 11, 161-226.

Wang, M. C., \& Zollers, N. J. (1990). Adaptive instruction: an alternative service delivery approach. Remedial and Special Education, 11, 7-21.

Washburne, C. W. (1925). Adapting the schools to individual differences. In G. M. Whipple (Hrsg.), The twenty-fourth yearbook of the national society for the study of education (S. 257-272). Chicago: University of Chicago Press.

Waxman, H.C., Wang, M.C., Anderson, K. A., \& Walberg, H. J. (1985). Adaptive education and student outcomes: a quantitative synthesis. Journal of Educational Research, 78, 228-236.

Waxman, H.C., Alford, B.L., \& Brown, D.B. (2013). Individualized instructions. In J. Hattie \& E.M. Anderman (Hrsg.), International guide to student achievement (S. 405-407). New York: Routledge.

Wellenreuther, M. (2008). Wieweit lösen individualisierende Methoden Probleme der Heterogenität in Schulklassen? Eine Diskussion anhand empirisch-experimenteller Forschung. In R. Lehberger \& U. Sandfuchs (Hrsg.), Schüler fallen auf. Heterogene Lerngruppen in Schule und Unterricht (S. 178-190). Bad Heilbrunn: Klinkhardt.

Wember, F. B. (2001). Adaptiver Unterricht. Sonderpädagogik, 31, 161-181.

Wiliam, D. (2010). The role of formative assessment in effective learning environments. In H. Dumont, D. Istance, \& F. Benavides (Hrsg.), The nature of learning. Using research to inspire practice (S. 135-159). Paris: OECD.

Winne, P.H. (2005). A perspective on state-of-the-art research on self-regulated learning. Instructional Science, 33, 559-565.

Winne, P. H., \& Perry, N.E. (2000). Measuring self-regulated learning. In M. Boekaerts, P. R. Pintrich, \& M. Zeidner (Hrsg.), Handbook of self-regulation (S. 531-566). San Diego: Academic Press.

Wirth, J., \& Leutner, D. (2008). Self-regulated learning as a competence. Implications of theoretical models for assessment methods. Zeitschrift für Psychologie, 216, 102-110.

Wischer, B. (2007). Heterogenität als komplexe Anforderung an das Lehrerhandeln. Eine kritische Betrachtung schulpädagogischer Erwartungen. In S. Boller (Hrsg.), Heterogenität in Schule und Unterricht. Handlungsansätze zum pädagogischen Umgang mit Vielfalt (S. 32-41). Weinheim: Beltz.

Wischer, B. (2012). Individuelle Förderung als Herausforderung für Schulentwicklung - Schultheoretische Perspektiven zu Konzepten und Fallstricken. In C. Solzbacher, S. Müller-Using, \& I. Doll (Hrsg.), Ressourcen stärken! Individuelle Förderung als Herausforderung für die Grundschule (S. 55-67). Köln: Link.

Wischer, B. (2014). Individuelle Förderung als neue Leitidee? Kritische Anmerkungen zu einer aktuellen Reformstrategie. In V. Eisenbraun \& S. Uhl (Hrsg.), Geschlecht und Vielfalt in Schule und Lehrerbildung (S. 163-176). Münster: Waxmann.

Wood, D., Bruner, J.S., \& Ross, G. (1976). The role of tutoring in problem solving. Journal of Child Psychology and Psychiatry, 17, 89-100. 
Wrase, M. (2015). Die Implementation des Rechts auf inklusive Schulbildung nach der UN-Behindertenrechtskonvention und ihre Evaluation aus rechtlicher Perspektive. In P. Kuhl, P. Stanat, B. LütjeKlose, C. Gresch, H. A. Pant, \& M. Prenzel (Hrsg.), Inklusion von Schülerinnen und Schülern mit sonderpädagogischem Förderbedarf in Schulleistungserhebungen (S. 41-74). Wiesbaden: Springer VS.

Zimmerman, B.J. (1990). Self-regulated learning and academic achievement: an overview. Educational Psychologist, 25, 3-17.

Zimmerman, B. J. (2000). Attaining self-regulation: a social cognitive perspective. In M. Boekaerts, P. R. Pintrich, \& M. Zeidner (Hrsg.), Handbook of self-regulation (S. 13-39). San Diego: Academic Press.

Zimmerman, B.J., \& Schunk, D.H. (1989). Self-regulated learning and academic achievement: theory, research, and practice. New York: Springer. 